\title{
Satzartige situative Ellipsen, ihre syntaktische und ihre semantische Repräsentation und ihre pragmatische Interpretation
}

\section{Summary}

The grammatically determined meaning of situation dependent ellipses like / Schnell einen Krankenwagen! / is indeterminate as compared to the semantic form of corresponding non elliptical sentences. To essume such an indeterminacy is only possible if one distinguishes the grammatically determined meaning from the utterance meaning. The seman tic indeterminacy of eituation dependent ellipses has its ayntectic pendant in the form of minimsl sentence structures containing empty categories, which are interpreted semantically as free variables. The let ter are replaced by specific context dependent entities only in the utterance meaning.

\section{Einführung}

Im Mittelpunkt des vorliegenden Aufsatzes steht die Besntwortung der folgenden drei Fragen: (1) Was ist unter satzartigen situativen Ellipsen zu ver'stehen? (2) Wie werden sie syntakt isch und semant isch repräsentiert und wie erfolgt ihre kontextuelle Interpretation? (3) W ws mucht ihre Spezifik gegenüber anderen ellipt ischen Ausdrücken aus?

Unter einer Ellipse wird allgemein ein Ausdruck veratanden, der in Vergleich zu einer möglichen vollständigen Entsprechung unvollständig ist. Die jeweilige Unvollständigkeit

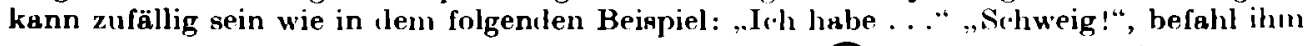
der Lehrer. Hier interessieren nur Ausdrürke wie / Den Hammer !/, / Eine Zigarette?/ und / Wunderbar!/. Ihre Unvollständigkeit kann auf das Wirken bestimmter Regeln zurückgeführt werden. Es werden dabei Regeln unterschieden, die grammatische strukturen erzeugen und Regeln, die die Verwendung sprachlicher Ausdrücke determinieren. Welche grammatischen und welche mußergrammat ist:hen strukturebenen hier zugrunde gelegt werden, soll im folgenden kurz skizziprt werden.

Es wird davon ausgegangen, daß eine illokutive Handlung $(1 H)$, als die die hier untersuchten Ellipsen verstanden werten, bestimmt ist durch ein lautliches oder gruphisithes Gebilde (ins), durch eine grammatische Struktur, durch eino Äußerungsbedeutung (e(Nv)), der eine bestimmte illokutive Funktion (IF) zugeordnet ist, und durih den kommunikativen Sinn (KS).

Die grammatische Struktur setzt sich zusammen uus der phonologischen, ler synt sktiochen und der semantischen Struktur, (pt, syn, ATT (PG)). Wie im dritten Kapitel noch ausgeführt werden wird, sind unter PG der propositionale Gehalt einer Äußerung und unter ATT der auf ihn bezogene Einstellungsoperator zu verstehen. Den Hintergrund der hier angestellten syntaktischen Úberlegungen bildet die Revidierte Erweiterte Standardtheorie (REST). Mit Bierwiscr (1987) wird hier in Ergänzung zu CномSкy (1981) und anderen als zusätzliche Strukturebene die semantische Form (SF) in die Grammutik uufgenommen. Die semant ische Form wird durch Regeln des konzeptuellen Kenntnissystems (CS) und des Systems (CA) interpretiert, das die in C'S realisierbaren und in der Grammatik repräsentierbaren Strukturbildungen als Objekte einer Einstellung oder einer Konfiguration von 
Einstellungen bewertet. Diese Regein nehmen Bezug auf den durch CS und CA determinierten Kontext ( $(\cdot \mathrm{cs} / \mathrm{CA})$ und erzeugen die ÄuBerungsbedeutung. Jene wird durch die Regeln des kommunikativen Kenntnissystems (KI) interpretiert. Dabei wirl zunächst in Ablı̈ngigkeit von dem jeweiligen Einstellungsoperator (ATT) der ÄuBenungsbedeutung eine illokutive Funktion (IF) zugeordnet. ${ }^{2}$ Indem die ÄuBerungsbedeutung mit der zugeordineten illokutiven Funktion suf den Interaktionskontext ( ct $_{1}$ ) bezogen wird, wird der kommunikative Sinn der illokutiven Handlung bestimmt. Bei der Bestimmung einer illokutiven Handlung wirken, wie oben deutlich wurde, mehrere Kenntnissysteme zusammen, die Grammatik (G), die die sprachliche Strukturbildung determiniert, das konzeptuelle Kennt nissystem (CS), das Einstellungskenntnissystem (CA) und das kommunikstive Kenntnissystem (KI). Im folgenilen Schens soll das eben Erläuterte zusammengefaBt werden. Die wasgerechten Pfeile symbolisieren Regeln, die jeweils zum Inventar eines bestimmten Kenntnissystems gehören. Die Zugehörigkeit wird im Schems durch geschweifte Klammern kenntlich gemarht. ${ }^{3}$

(1) $\mathrm{IH}=\mathrm{ins} \underbrace{\rightarrow \mathrm{pt} \rightarrow \mathrm{Byn} \rightarrow \mathrm{ATT}(\mathrm{PG})}_{\mathrm{G}} \underbrace{-\mathrm{ct} \mathrm{cs} / \mathrm{CA}_{\mathrm{A}} \rightarrow \mathrm{e}(\mathrm{sv})}_{\mathrm{CS} \mathrm{CA}} \rightarrow \underbrace{\mathrm{IF}-\mathrm{ct}_{\mathbf{I}} \rightarrow \mathrm{KS}}_{\mathrm{KI}}$

Wie oben erwähnt, gibt es unvollständige Ausdrūcke wie (2) und (3), deren Unvollstāndigkeit auf die Grammatik zurückführbar ist.

(2) Peter ist größer als Paul (groB ist).

(3) Peter iBt Eis und Paul (iBt) Kuchen.

Sie sind, wie in Abschnitt 2.4. gezeigt werden wird, durch grammatische Regeln, die auf den aprachlichen Kontext Bezug nehmen, interpretierbar. Anders verhält es aich bei den hier als situstive Ellipsen bezeichneten unvolletändigen Ausdrūcken wie (4), (5) und (6).6

(4) Den Hammer!

(5) Eine Zigarette?

(6) Jetzt aber schnell!

Diese Ausdrücke sind durch die Gramnatik allein nieht hinreichend interpretierbar. Ihre Unvollständigkeit ist in erheblichem MaBe situativ (auBersprachlich) bedingt. Wie elliptische Aussirūeke (4), (5) und (6) syntaktisch und semantisch repräsentiert werden und wie ihre Äußerungsbedeutung und ihr komnunikstiver Sinn ermittelt werden, macht den Inhalt dieses Aufsat zes aus. Die Repräsentstion der syntaktischen und semantischen Struktur jener Ausdrücke sowie deren Aủerungsbedeutung sind deshelb interessent, weil hier davon ausgegangen wird, daB die semantische Struktur von Ellipsen wie (4), (5) und (6) im Vergleich zu sprachlich gestützten Ellipsen wie (2) und (3) und möglichen vollständigen Entsprechungen unbestimmt ist. ${ }^{5}$ Ihre sementische Form ist insofern unspezifiziert, sls zur Eingrenzung ihres Referenten im Gegensatz zu (2) und (3) und zu etwaigen vollständigen Entsprechungen nur ein Minimum sprachlich gegeben ist. Die semantische Unbestimmtheit resultiert aus dem oben unter (1) dargestellten Prinzip der Laut-Bedeutungs-Zuordnung. Die Satzbedeutung (semantische Form eines Satzes) ist danach die direkte Interpretstion der syntaktischen Struktur. Die Äußerungsbedeutung hingegen ergibt sich ans dem Bezug der Satzbedeutung auf einen bestimmten Kontext. Die Unterscheidung von Satzhedeutung und ÄuBerungsbedeutung wendet die Gefahr ab, bei einer linguistischen Analyse Beispielsätze mit naheliegenden Interpretationen zu versehen, indem man typische Kontext e hinzudenkt und sonit die Strukturbetrachtung interpretatorisch überlagert. Eine derartige strukturelle Uberfrachtung ist beispielswei se dann der Fall, wenn für die

1 Vgl. BIERWTSCh (1983), LANG (1985).

2 Vgl. МотsCh/Pasch (1987).

3 Ebila.

4 Neben jenen unvollständigen Ausdrücken, die durch die Grammatik interpretierber sind und solchen wie (4), (5) und (6), die in erheblichem Maße durch den situativen Kontext determiniert sind, ist eine dritte Gruppe anzunehmen. Gemeint sind dislogische Ellipsen, die zwar wie die erste Gruppe durch den Bezug auf den sprachlichen Kontext interpretierbar sind, bei denen jedoch zusätzlich zu den grammatischen Regeln bestimmte 'dialogisehe' Regeln anzunehmen sind.

5 Vgl. SHOPEN (1972 und 1973) und ZrMMERMANN (1981). 
Äußerung Den Hammer/ uls Bedeutung 'Hörer gibu sprecher den Hammer' interpretiert werden würde. Die ayntaktiache Struktur von Den Hammer! enthält aber nur eine Nominalphrase im Akkusativ, die eine derartige semant ische Interpretation nicht zuläßt.

Strukturelle Utberspezifiziertheit tritt auch dann auf, wenn wie in HEIdolpH (1981) angenommen wird, daBsituative Ellipsen wie (4), (5) und (6) eine mit lexikalisehen Einheiten vollständig besetzte Tiefenstruktur aufweisen, in der durch bestimmte Transformationen Konstituenten getilgt werden. WhLLAms (1977) und SHOPEN (1972 und 1973) zeigen überzeugend, daß diese transformstionalistische Auffassung dem hier \%ugrunde gelegten Prinzip der Laut-Bedeutunga-Zuordnung nicht gerecht wird.6

Aus dem obigen Prinzip ist der für die syntakt ische und semant ische Beschreibung situs. tiver Ellipeen nicht zu ignorierende methodische Grundsat $z$ ableitbar :

(7) Die semantische Reprägentation eines Ausdrucks S darf nur soviel an semantischer Information enthalten wie durch die grammatische Strukturbildung in $s$ vorgezeichnet ist.

Die grammatische Struktur ist, wie unten deutlich wird, determiniert durch Lexikoneinheiten mit ihrer internen Struktur, durch syntakt isch realisierte kombinatorische Eigenschaften und die damit agierenden intonatorischen und prosodischen Kennzeichnungen.

Geht man duvon aus, daB die semantische Struktur situativer Ellipsen ziemlich unspe. zifiziert ist, sieht man sich mit drei Fragen konfrontiert. Erstens, wie wird die semantische Unbestimmtheit situativer Ellipsen repräsentiert? Zweitens, wie ist der Input für derartig unbestimmte semant ische Formen, also die entsprechende gyntakt ische Struktur repräsentiert? Und drittens, wie werden unbest immte semantische Strukturen interpretiert? Die Beantwortung der zweiten Frage wird im folgenden Kapitel versucht.

\section{Die syntaktische Reprāsentation situativer Ellipsen}

\subsection{Einführende Bemerkungen}

Das zweite Kapitel beschäftigt sich mit der syntaktischen Beschreibung von unvollständigen Ausdrücken. Der Schwerpunkt liegt dabei auf der Beschreibung der syntaktischen Struktur von situativen Ellipsen und deren Abgrenzung von ausschließlich grammatisch determinierten Ellipsen.

Wir gehen davon aus, daß die syntaktische Struktur modular ist. Sie besteht aus der Tiefenstruktur (D-Struktur), aus der 'surface structure' (S-Struktur), der Oberflächenstruktur (O-Struktur) und der Logischen Struktur (LF). Die D-Struktur wird, wie noch zu beschreiben ist, durch Formationsregeln erzeugt und von den Regeln der S-Strukturebene interpretiert.` Die resultierende S-Struktur dient zum einen als Eingabe für Regeln der LF-Komponente und zum anderen als Input für die Regeln der OS-Komponente. Die Logische Form wird durch semantische Regeln interpretiert. Ihre semantische Interpretation (SF) ist als vermittelnde Repräsentationsebene für die Korrelierung von Logischer Form und konzeptuellen Struktureinheiten (CS) anzusehen. ${ }^{8}$ Phonologische Regeln schließlich bilden die Oberflächenstruktur auf die Phonologische Form (PF) ab.

Charakteristisch für die D-Strukturebene sind die Formationsregeln. In Anlehnung

6 Vgl. Grochowski (1985) und SchwaBe (1987).

i Vgl. Chomsky (1981), Bierwisch (1987) und Zimmermasn (1985).

6 Vgl. Bienutsch $(1980,1981,1983,1985$ und 1987). 
an JACKEN dofF (1977) und ZnMmermanN (1985 und 1987), BLERWISCh (1985) und ('ном⿰ку (1985) werden hier die folgenden Regeln angenommen:9

(8)
(i) $\mathrm{X}^{1} \rightarrow \mathrm{Y} \div \mathrm{X}^{n}$
(ii) $\mathrm{X} * \rightarrow \mathrm{Y}^{*} \mathrm{X}^{1}$
(iii) $\mathrm{X}^{\mathrm{i}} \rightarrow \mathrm{Y}^{-1} \cdot \mathrm{X}^{\mathrm{i}}$
(iv) $\mathrm{X}^{\mathrm{i}} \rightarrow \mathrm{e}$

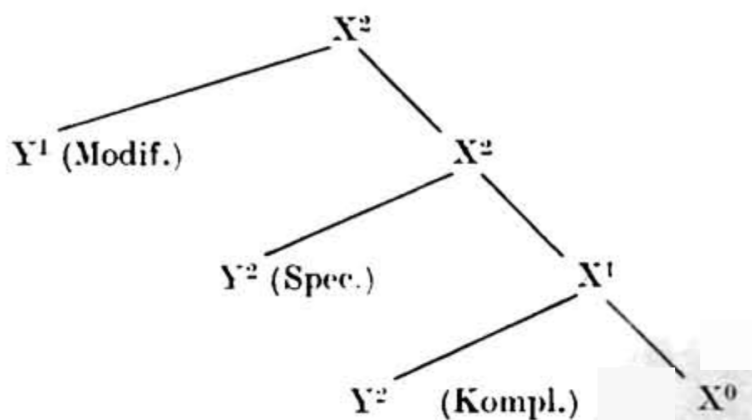

(Kompl.)

Die Variablen $\mathrm{X}$ und $\mathrm{Y}$ stehen für syntaktische Kategorien, der Zahlenindex signalisiert die jeweilige Projektionsstufe, * symbolisiert das n-malige Vorkommen der betreffenden Kategorie und ' $\rightarrow$ ' meint 'direkte Dominanz'. Die Kategorie $\mathbf{X}^{\mathrm{i}-1}$ ist als der Kopf von $\mathbf{X}^{\mathbf{i}}$ anzusehen. Die Regel (i) erzeugt Strukturen, die Komplemente ( $\left.{ }^{2}\right)$ enthalten, (ii) generiert Syntagmen mit Spezifikatoren, und (iii) führt Modifikatoren ein. ${ }^{10}$ Für die Kategorievariable $\mathrm{X}$ beziehungsweise $\mathrm{Y}$ werden-Kategorienbezeichnungen wie $\mathrm{V}, \mathrm{N}, \mathrm{Adj}$, Adv, P, Spec, INFL und COMP beziehungsweise $\mathrm{S}$ eingesetzt." Entsprechend dem Bildungsschema (8) und СномSкY (1985) kann man beispielsweise für einen Satz die Struktur (9) annehmen (siehe S. 596).

Das Schema zeigt, daß der Satz die maximale Projektion des Complementizers COMP ist. Ohne dieses Schema weiter diskutieren zu wollen, sei darauf verwiesen, da B der Status der von V² unmittelbar dominierten Kat egorie X² ziemlich unklar ist. Ihre Existenz beruht darauf, daß jede maximale Projektion entsprechend (8) (ii) einen Spezifikator dominiert.

Die nach dem Schema (8) erzeugten D-Strukturen werden mit Hilfe von Transformationen ( $T_{1}$-Regeln) auf $\mathrm{S}$-Strukturen abgebildet. Durch $T_{1}$-Regeln können Konstituenten $K_{1}$ unter Zurücklassung einer Spur $e_{i}$ auf bestimmte Positionen verschoben werden. Jene S-Strukturen unterliegen Wohlgeformtheitsbedingungen, auf die hier nicht weiter eingegangen werden kann. Außer jenen $T_{1}$-Regeln ist für die S-Struktur-Ebene die Kasuszuweisung relevant. Auf sie wird an gegebener Stelle zurückzukommen sein.

Die Regeln der Logischen-Form-Ebene interpretieren die S-Struktur und erzeugen damit den Input für die semantische Interpretation. Quantorenverschiebung, die Kontrolle von Pronomen und von bestimmten leeren Kategorien sind als Beispiele

"Die Wahl des hier verwendeten, von Chомsкy (1985) übernommenen, X-Sehemas kann in diesem Rahmen nicht weiter gerechtfertigt werden. Wichtig in bezug auf das Anliegen dieses Aufsutzes ist, daß es sich bei den verwendeten Formationsregeln um Regeln der $X$-Theorie handelt. Die bedingte Austauschbarkeit jener Regeln zeigt sich darin, da $B$ ich in meiner Dissertation (Schwabe (1987)) das von Zrmmersasx (1985) entworfene $\mathrm{X}$-Schema verwendet habe.

10 Nach Brenwrsch ist dio maximale Projektion von Modifikatoren $\mathrm{X}^{\prime}$, da diese keine Spezifikstoren aufweisen können.

11 Nhch ZimmermanN (1985) könnten für $\mathrm{X}$ bzw. Y Wortklassenmerkınale eingesetz.t werden. Da eine Diskussion über die alternative Ersetzung von $\mathbf{X}$ oder $\mathbf{Y}$ für die Thematik dieses Aufsat zes irrelevant ist, sollen die bekannteren Bezeichnungen verwendet werden. 
(9)

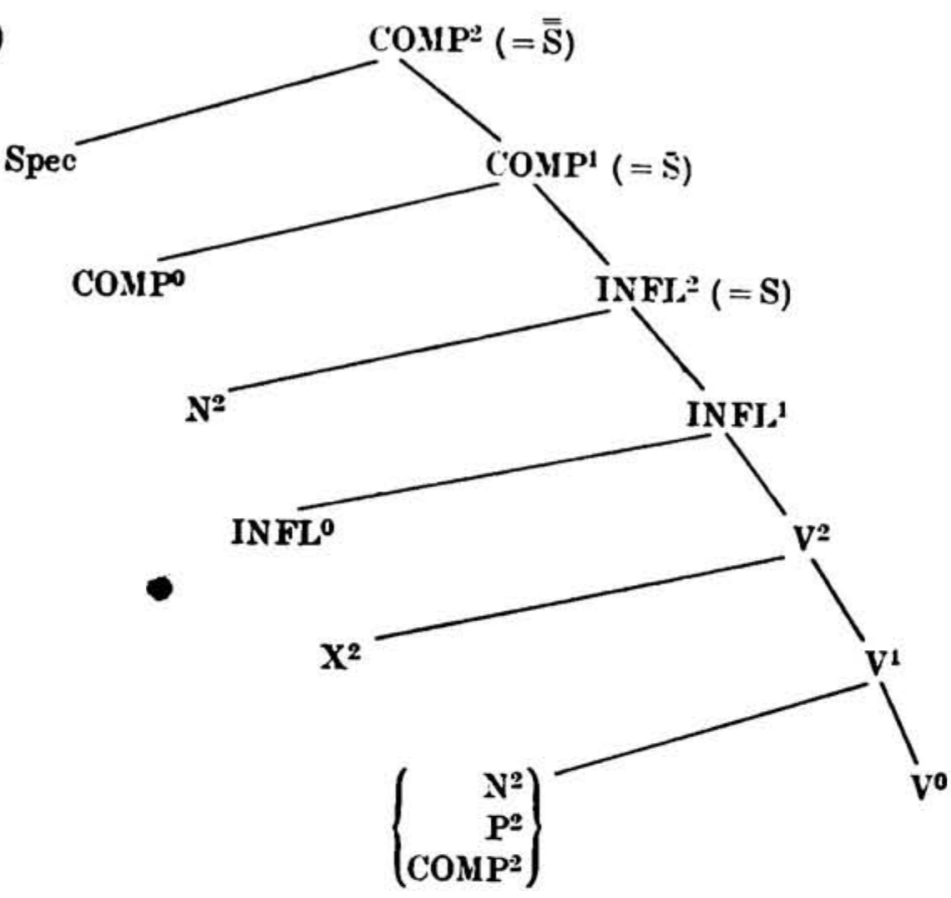

für die Regeln der LF-Komponente anzusehen. Weiterhin gehen wir mit CHomsky (1982) und Bierwisch (1987) davon aus, daB in LF bestimmte syntaktische Positionen $\theta$-markiert werden. Die lexikalisthen Einheiten enthalten neben ihrer phonologischen Repräsentation, der Angabe der syntaktischen Kategorie und gegebenenfalls der Subkategorisierungsmerkmale eine semantische Form. Diese giht auBer der internen semantischen Struktur das $\Theta$-Raster an. Jas syntaktisch relevante, semantische $\boldsymbol{\theta}$-Raster besteht aus Lambdaabstraktoren, die bestimmte Variablen in der internen semantischen Form des betreffenden Lexems binden. Die Lambdaabstraktoren werden von BıERwisch als thematische Rollen aufgefaßt. Diese $\boldsymbol{\theta}$-Rollen werden in der Logischen Form an bestimmte syntaktische Positionen vergeben. Welche syntaktische Position welche $\theta$-Rolle zugewiesen bekommt, wird durch die entsprechende Rollen zuweisende lexikalische Einheit dadurch festgelegt, daß ein Index einer $\theta$-Rolle angibt, welche syntaktische Position als Rollenträger in Frage kommt.

\subsection{Zugänge}

Es wird hier intuitiv davon ausgegangen, da $B$ es sich bei Ausdrückell wie (10) Schnell den Hammer!

(11) Vor dir!

(12) Eine Zigarette?

um satzartige Ausdrücke handelt. Sieht man von dieser Intuition zunächst ab, ist zu fragen, wie die syntaktischen D-Strukturen jener Ausdrücke in Abhängigkeit von dem Schema (8) erzeugt werden sollen. Zwei Alternativen sind denkbar. Erstens, es mu $B$ in der Grammatik kein einheitliches Startsymbol COMP² geben. Das heißt, da $B$ die Ausdrücke (10) bis (12) anders als vollständige Sätze nicht COMP 2 bzw. 
$\overline{\mathrm{S}}$ sondern im Fall von (10) $\mathrm{V}^{2}$, in bezug auf (11) $\mathrm{P}=$ und im Fall von (12) $\mathrm{N}$ - als oberste Knoten aufweisen wïrden.

Die Konsequenz dieser iibrigens von Sнореx (1972) vertretenen Auffassung ist, daB die Grammatik beliebige sprachliche Ausdrücke, die Sätze und Fragmente sein können, erzeugt. Da von dieser Annahme noch des öfteren die Rede sein wird, soll sie abkürzend die a-Hypothese genannt werden. Gegen die a-Hypothese spricht, daß sowohl bei (10) als auch bei (12) der Kasus der Nominalphrase nicht erklärt werden kann, da keine Konfiguration zur strukturellen Zuweisung des Kasus gegeben ist. Desweiteren ist intuitiv nicht einsehbar, da B (10) eine nicht strukturierte Kette von maximalen Projektionen sein soll. Gegen die a-Hypothese sprechen auch eingebettete Sätze wie (13) und (14), für die gemäB der a-Hypothese gelten müßte, $\mathrm{da} B$ sie keine Struktur aufweisen. Und es stellt sich die schwer zu beantwortende Frage, wie die kursiven Ausdrücke in (13) und (14) erzeugt werden sollen.

(13) In der Wüste waren die Kamele der Beduinen früher genauso schnell wie die Autos dort heute.

(14) Er kennt seinen Freund besser, als ich meinen.

Die eben aufgezeigten Probleme, die auch im Rahmen der a-Hypothese schwerlich gelöst werden können, lassen sich durch die zweite Alternative, wir nennen sie bHypothese, zufriedenstellender klären. Bei der b-Hypothese gehen wir davon aus, daß alle durch die Grammatik erzeugten satzartigen Ausdrücke wie (10), (11) und (12) COMP2 als einheitliches Startsymbol aufweisen. Die durch die Formationsregeln erzeugten terminalen Ketten können dabei leere Ketten enthalten. Was unter diesen leeren Ketten zu verstehen ist, wird unten erklärt.

\subsection{Ein Weg zur syntaktischen Repräsentation situativer Ellipsen}

Es wurde bereits angedeutet, daB situative Ellipsen wie (10), (11) und (12) Sätze sind. Diese Annahme kann folgendermaßen begründet werden. Frstens, geht man davon aus, daß der Kasus von bestimmten Nominalphrasen strukturell bedingt ist, muB eine entsprechende Struktur vorausgesetzt werden. Da es Ellipsen wie Ein Flugzeug! gibt, bei denen in einer Interpretation die Nominalphrase im Nominativ steht, ist als oberster Knoten mindestens INFL?, der das Subjekt unmittelbar dominiert, anzusehen. Zweitens, es ist davon auszugehen, da B auch in situativen Ellipsen der Satzmodus, wenn auch in den meisten Fällen ganz minimal, nur durch die Intonation, realisiert wird. Da angenommen wird, da B COMP0 sozusagen die satzmodusrelevanten syntaktischen Gegebenheiten dominiert, muB COMP2 bzw. $\mathbf{S}$ als oberster Knoten für situative Ellipsen angesehen werden.12 Vor dem Hintergrund dieser Argumente wird davon ausgegangen, daB die D-Struktur zumindest von Ellipsen wie (10), (11) und (12) COMP2 als obersten Knoten aufweist.

Aus dem Regelschema (8) geht hervor, daB jede Kategorie $\mathbf{X}^{i}$ zu einer leeren Kette expandiert werden kann. Diese leere Kette ist von $\mathbf{X}^{i}$ ausschlieBlich dominiert. Leere Kategorien sind durch ihre kategoriellen Merkmale und durch ihre Projektionsstufe charakterisiert. Sie können, unter Voraussetzung der b-Hypothese, durch (8) erzeugt oder nach Cномsky (1981) Spuren von Trangformationen sein. Bei letzteren kann es sich um Transformationen $T_{1}$ der S-Struktur-Ebene oder um Transformationen $\mathrm{T}_{2}$ der LF-Ebene handeln. AuBer den eben genannten zwei Typen von leeren Kate-

12 Zum Verhältnis von COMP und Satzmodus vgl. LaNa/Pascr (1986). 
gorien, den durch (8) erzeugten und den Spuren, werden auch phonologisch leere pro-Elemente als leere Kategorien angesehen. Es wird die Auffassung vertreten, da B diese pro-Elemente Lexikoneinheiten sind. Sie sind bis auf die fehlenden phonologischen Merkmale genauso wie nicht leere Lexikoneinheiten strukturiert. Das könnte in bezug auf die Erzeugung von situativen Ellipsen die alternative Annahme provozieren, da $B$ unter bestimmten Bedingungen $X^{0}$ durch die lexikalische Einheit pro erssetzt wird. Auf die Konsequenzen dieser Annahme, daB für das Lexikon zu befindem ist, welche und wieviel pro-Elemente es enthält, und welche Wohlgeformt heitsbedingungen für pro-haltige Strukturen gelten, wird in Schwabe (1987) eingegangen. Wir gehen, wie bereits erwähnt, davon aus, da $B \mathrm{X}^{\mathrm{i}}$ zu e expandiert werden kann. Gegeben sind dadurch für die leere Kategorie die kategorialen Merkmale und die Projektionssitufe. Wie in Kapitel drei deut lich werden wird, interpretieren semantische Regeln ein Minimum von semantischer Struktur für jene leeren Kategorien.

Zur Veranschaulichung der hier verfolgten Darstellung der D-Struktur situa:tiver Ellipsen soll die D-Strubturrepräsentation (15) der situativen Ellipse Den Hamimer! dienen. ${ }^{13}$

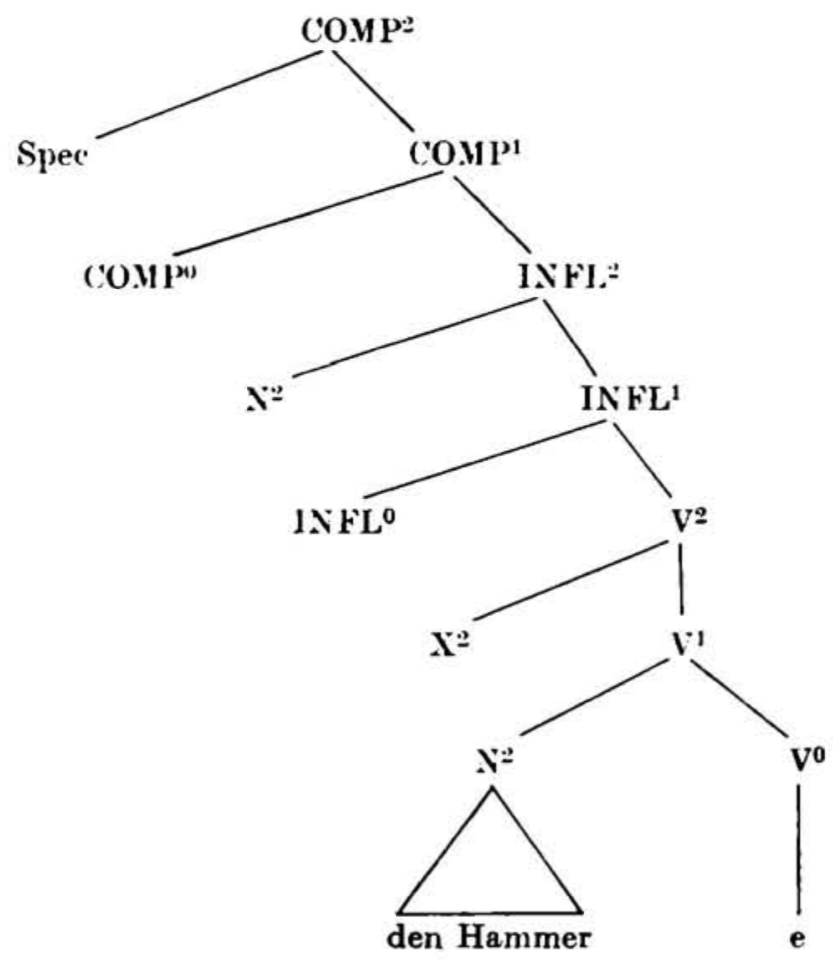

Für die durch (8) erzeugten Strukturen mit leeren Kategorien obne Antezedenten existieren auf den einzelnen Strukturebenen bestimmte Beschränkungen.14 Da hier

13 Die Besetzung von Spec, des Complementizers (COMP ${ }^{0}$ ) und von INFL ${ }^{0}$ wird vorläufig noch offen gelassen.

14 Unter einem Antezedenten wird hier eine nicht leere Kategorie verstanden, die süch in einer Struktur befindet, die parallel zu der strukturiert ist, in der sich die leere Kategorie befindet. In dem Beispiel Peter $i \beta t_{1}$ Fisch und Paul $\left[V_{i}^{0} \theta\right]$ Fleisch ist $i \beta t_{1}$ der Antezedent von $\left[V_{i}^{0}\right.$ e]. 
nicht das Ziel verfolgt werden soll, sie alle zu erkennen und zu präsentieren, sollen nur einige genannt werden. Soweit es möglich ist, werden sie in den für sie relevanten Abschnitten behandelt.

Generell ist zu bemerken, da B entsprechend den in der Einleitung unter (7) genannten (inundsatz der Laut-Bedeutungs-Zuordnung nicht mehr leere Kategorien erzeugt werden können als sprachlich indiziert sind. Deshalb kann es beispielsweise keine kontext unabhängigen elliptischen Ausdrücke geben, die fakultative leere Kategorien wie $\left[\mathrm{x}_{1} \mathrm{e}\right]$ mit Modifikatorfunktion enthalten. Mit anderen Worten, es können nur strukturnotwendige Kategorien leer sein. Das sind in bezug auf das Schema (9) die Kategorien COMP ${ }^{i}$, Spec. ${ }^{i}$, INFL', Vi, die von INFL 2 unmittelbar dominierte N 2 , Köpfe von Kategorien und Komplemente in bestimmten syntaktischen Konfigurationen.

Das bewuBte Prinzip bedingt zudem, da $B$ eine leere Kategorie cine nicht-leere Kategorie $\cdot-k o m n a n d i e r e n ~ m u B .15$ Dadurch werden Kategorien, die nur leere Kategorien dominieren, ausgeschlossen.

Im folgenden soll dargelegt werden, ob die Kategorien INFL $^{i}$ und $\mathbf{V}^{\mathbf{i}}$ als leere Kategorien ohne Antezedenten in syntaktisch wohlgeformten Strukturen vorkommen können. ${ }^{16.17}$

INFL:

(16) [Kontext: $B$ kommt mit froher Miene aus einer Prüfung. $A$ und $B$ hatten zuvor Bedenken, ob $B$ die Prüfung bestehen würde.]

(i) A: Wirklich?

(ii) $\mathrm{LF}: \mathrm{COMP}_{\mathrm{COMP}^{2}} \mathrm{Spec}\left[\mathrm{COMP}_{1} \operatorname{COMP}^{0}\left[\mathrm{INFL}_{2}{ }^{\text {wirklich }}\left[\mathrm{INFL}_{2}{ }^{\mathrm{e}}\right]\right]\right]^{18}$

INFL 1

(17) [Kontext: $A$ und B warten auf ein Flugzeug. Sie blicken zum Himmel, weil dort ein Geräusch zu hören ist.]

(i) A: Ein Flugzeug!

(ii) LF: [ $\mathrm{COMP}_{2} \mathrm{Spec}\left[\mathrm{COMP}_{1} \mathrm{COMP}^{0}\right.$ [INFL $^{2}$ ein Flugzeug [ INFL $\left.\left.\left.{ }^{\mathrm{e}}\right]\right]_{]}\right]$

$\mathrm{Zu}$ bemerken ist, daB die Nominalphrase ein Flugzeug in (17) (i) insofern mehrdeutig ist, als sie auch als Nominalphrase im Akkusativ interpretiert werden könnte. Die syntaktische Repräsentation würde dann der von (19) entsprechen.

INFL $0, V^{2}$

(18) [Kontext: $A$ zieht sich an. $B$ ist das Anziehen von $A$ zu langsam.]

(i) B: Schneller!

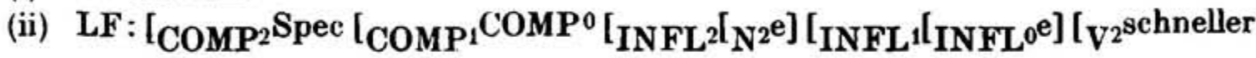
$\left[V_{2}\right.$ e $\left.\left.\left.\left.]\right]\right]\right]\right]$

15 Unter X c-kommandiert $\mathrm{Y}$ ist nach Сномвку (1981) zu verstehen, da B der nächst höhere verzweigende $\mathrm{Knoten}$ von $\mathrm{X} \mathrm{Y}$ dominiert.

${ }^{16}$ Die Kategorie COMP ${ }^{1}$ bleibt aufgrund des diesbezüglichen Forschungsstandes hier unanalysiert.

17 Auf das Vorkommen von leeren $\mathrm{N}^{\mathrm{i}}, \mathrm{Adj}^{\prime}, \mathbf{A d v}^{\mathbf{i}}$ und $\mathrm{P}^{\mathbf{l}}$ wird in Schware (1987) eingegangen.

18 Es sei in diesem Zusammenhang darauf verwiesen, daß im Rahmen dieses Aufatzes auf die Regeln, die die D-Struktur auf die S-Struktur und die S-Struktur auf die Logische Form abbilden, nicht weiter eingegangen werden kann. Vorab wird davon susgegangen, daß sich bei den genannten Beispielen jene Strukturen nicht weiter unterscheiden, so daB nur die Logische Form, die dureh semant ische Regeln interpretiert wird, angegeben wird. 
In (18) (ii) und den folgenden Beispielen wird deutlich, da B INFL0 immer dann leer sein muB, wenn es keine lexikalische Finheit V c-kommandiert. Es sei darauf verwiesen, da B der Modifikator schneller hier in erster Festlegung $V=$ modifiziert. Da das Subjekt zumindest für das Deutsche obligatorisch ist, wird es hier durch die leere Kategoric $N^{2}$ repräsentiert.

I0

(19) [Kontext: $A$ arbeitet, und $B$ hilft dabei. Auf dem T'isch liegt ein Hammer.]

(i) A: Den Hammer!

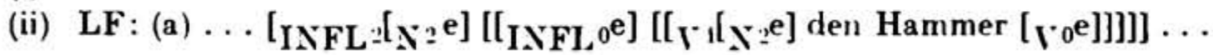
(b) $\ldots\left[\left[_{\gamma,}\right.\right.$ den Hammer $\left.\left[\gamma_{0} e\right]\right] \ldots$

Die Repräsentationen (a) und (b) unterscheiden sich darin, da B (a) zwei Komplemente und (b) nur ein Komplement aufweist. $\mathrm{DaB}$ (a) neben dem lexikalisch realisierten Komplement den Hammer ein leeres Komplement aufweist, ist im Zusammenhang mit den Akkusativkasus von den Hammer zu sehen. Da sowohl in (a) als auch in (b) kein Verb enthalten ist, das der Nominalphrase einen Kasus zuweisen könnte, ist der Akkusativ von den Hammer strukturell bedingt.

Es wird davon ausgegangen, da B bei einer Nominalphrase, die ein Komplement von $V^{0}$ ist, der Akkusativ strukturell bedingt ist, wenn jene Nominalphrase das einzige Komplement von $V^{0}$ ist, oder wenn, weist $V^{0}$ zwei nominale Komplemente auf, die linear gesehen $\mathrm{V}^{\circ} \mathrm{am}$ nächsten steht, und wenn durch die von $\mathrm{V}^{0}$ dominierte lexikalische Einheit kein anderer Kasus gefordert wird. Weiterhin wird angenommen, da $B$ bei einem Komplement von $V^{0}$ der Dativ strukturell bedingt ist, wenn es die linke Kokonstituente eines Komplements im Akkusativ ist, und ihm durch eine von Vo dominierte lexikalische Einheit kein anderer Kasus zugewiesen wird. Die eben genannten strukturellen Bedingungen treffen auf die Beispiele (19) (a) und (b) und (20) zu.

(20) [Kontext: Widmung in einem Buch]

Meiner Mutter

LF: . . [ [ $\mathrm{V}$, meiner Mutter $\left[\mathrm{N}_{2} \mathrm{e}\right]\left[_{\mathrm{V}_{0} \mathrm{e}} \mathrm{e}\right] \ldots$

Für die S-Strukturebene, auf der die Kasuszuweisung stattfinden soll, kann demnach folgende Bedingung angegeben werden. ${ }^{19}$

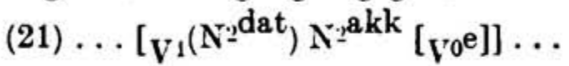

Durch die Bedingung (21) werden Ausdrücke wie beispielsweise Des Hammers! und An meine Mutter. als nicht wohlgeformt ausgefiltert, setzt man voraus, da B es sich bei der Nominalphrase und der Präpositionalphrase um Objekte mit einem inhärenten Kasus handelt.

Die Beispiele (16) bis (20) zeigen, da ß bestimmte situative Ellipsen als eine minimale Satzstruktur repräsentiert, sind. Die in den Strukturen enthaltenen leeren Kategorien weisen keinen Antezedenten auf. Diese sprachlich bedingten, minimalen Strukturen entsprechen dem eingangs erwähnten Prinzip der Laut-Bedeutungs-Zuordnung, da B in einer syntaktischen Struktur nicht mehr repräsentiert sein darf, als sprachlich indiziert ist.

Nun einige Bemerkungen zu der Logischen Form der aufgeführten Beispiele, spe-

${ }^{19} \mathrm{DaB}$ in (17) der Nominativ von ein Flugzeug ebenfalls strukturell bedingt ist, soll, da es keinen anderen Subjektkasus gibt, keine weitere Erwähnung finden. 
ziell zu der $\Theta$-Markierung. Wic bereits bemerkt, markiert in einer vollständigen Logischen Form eines Satzes die von $V^{\prime \prime}$ dominierte lexikalische Einheit aufgrund ihres $\Theta$-Rasters die jeweiligen Argumentpositionen. Als Argumente kommen dabei nur die Komplemente und das Subjekt in Frage. In bezug auf die Iogische Form von situativen Ellipsen ist es klar, da B ein Komplement und ein Subjekt nicht von einer leeren Vo $\Theta$-markiert werden können. Ich gehe davon aus, daB die betreffenden Komplementpositionen und die Subjektpositionen aufgrund ihrer syntaktischen Position als Argument position identifiziert werden. Diese Annahme ist damit zu rechtfertigen, da B in vollständigen Strukturen jedes Komplement $\theta$-markiert ist, und da $B$ das Subjekt nur dann nicht $\theta$-markiert ist, wenn es durch eine lexikalische Einheit so gefordert wird. Da die Beispiele (16) bis (20) keine Lexikoneinheiten $V$ enthalten, die die $\boldsymbol{\theta}$-Markierung der Subjekt position verbieten könnten, sind alle in den Beispielen enthaltenen Komplement- und Subjektpositionen als Argumentpositionen anzusshen.

\subsection{Die Spezifik situativer Ellipsen im Vergleich zu grammatisch determinierten El- lipsen}

Wie bereits bemerkt, stellt die syntaktische Beschreibung von unvollständigen Ausdriicken ohne sprachlichen Kontext einen Schwerpunkt dieses Aufsatzes dar. Ihre Tiefenstruktur (D-Struktur) wird in der in 2.3. erläuterten Weise erzeugt. Ihre Interpretamente auf der S-Strukturebene und auf der LF-Ebene enthalten leere Kategorien, die keinen Antezedenten aufweisen. Da bei ihrer semantischen Interpretation kein sprachlicher Kontext zur Verfügung steht, enthalten sie, wie im dritten Kapitel deutlich werden wird, nur eine minimale semantische Charakterisierung. Ihre konzeptuelle Interpretation ist deshalb kaum sprachlich determiniert, вo daB für die Außerungsbedeutung der minimal charakterisierten semantischen Einheiten ein ziemlich großer Variationsspielraum gegeben ist. Da aufgrund einer bestimmten Bedingung die konzeptuelle Belegung dieser semantischen Einheiten nur durch eine Gegebenheit des situativen Kontextes erfolgen kann, werden unvollständige Ausdrücke ohne sprachlichen Kontext als situative Ellipsen bezeichnet. Die eben genannte minimale semantische Charakterisierung des Interpretationsresultats bestimmter syntaktisch leerer Kategorien kann als wesentlicher Unterschied von situativen und anderen elliptischen Ausdrücken angesehen werden. Bei letzteren handelt es sich um Ausdrücke, die einen sprachlichen Kontext aufweisen. Als Beispiel dafür sind Komplementsätze von Komparativ- und Ãquativkonstruktionen, 'Gapping' und Repliken anzusehen. Bei Komplementsätzen und 'Gapping' ist als der sprachliche Kontext das erste Konjunkt beziehungsweise der Matrixatz anzusehen. Bei Repliken ist es der Vorgängersatz. Warum bei kontextabhängigen Ellipsen die leeren syntaktischen Kategorien im Gegensatz zu situativen Ellipsen eine reichere semantische Repräsentation aufweisen, soll im folgenden skizzenhaft dargelegt werden. 20

In bezug auf die Erzeugung von kontextabhängigen Ellipsen kann auch alternativ von der a- und der b-Hypothese ausgegangen werden. Im Rahmen der a-Hypothese ist für Repliken wie (22), Komplementsätze wie (23) und Konjunkte wie (24) eine D-Struktur anzunehmen, die syntaktisch nicht als Satz kategorisierbar ist.

20 In folgenden wird für Ellipsen, die mit dem Bezug auf ihren sprachlichen Kontext interpretiert werden, auch die Redeweise 'kontextabhängige' Ellipsen oder 'kontextuelle Ellipsen' verwendet.

40 Z. Phonetik (ZPSK) 41 (1988) 5 
(22) Wessen gedenkt Ibr? Des Bischofs.

(23) Der Tisch ist höher als breit.

(24) Peter schreibt seinem Vater und Paula ihrer Mutter.

BIERWISCB (1987), der sjch unter anderem mit der syntakt ischen Beschreibung von Komplementsätzen in Komparativkonstruktionen wie (23) beschäftigt, sieht jene in der Oberflächenstruktur, in der D-Struktur und in der S-Struktur als fragmentarische Komplemente an. Er schlägt vor, da B Regelı der LF-Ebene aus ihnen Satzstrukturen konstruieren. Die Endketten dieser Strukturen enthalten leere Ketten, die keine phonologische Form, aber eine semantische Repräsentation aufweisen. Die lceren Kategorien kommen somit nicht durch lexikalische Einsetzung, sondern durch syntaktische Projektion zustande. Ihre semantische Form wird durch Elemente des Matrixsatres determiniert, zu dem das Adjektiv gehört, dessen Gradangabe das Gradkomplement verlangt. Der Inhalt weiterer Forschungen könnte sein, ähnliche Parallelisierungsregeln für Repliken wie (22), Konjunktreduktionen wie (24) und andere in der Oberfächenstruktur unvollständige Sätze zu formulieren. Voraussetzung dafür ist aber, $\mathrm{da} B$ die a-Hy pothese zugrunde gelegt wird. Ob diese vorbehalt los angenommen werden kann, wurde in Abschnitt 2.2. erörtert.

Die b-Hypothese ging davon aus, da B satzartige Ausdrücke durch die Grammatik in der D-Struktur-Ebene als Sätze erzeugt werden. Ihre Endketten enthalten leere Kategorien. In bezug auf den Charakter dieser leeren Kategorien können zwei alternative Annahmen verfolgt werden. Frstens, es handelt sich um leere pro-Elemente, um lexikalische Einheiten, die eine semantische Form, aber keine phonologische Form aufweisen. Ausgenommen davon sind leere Kategorien, die Platzhalter in der D-Struktur und Spuren in der S-Struktur sind. Für Strukturen mit derartigen leeren pro-Elementen könnte die oben erwähnte Parallelisierungsregel wie auch andere als Filter umformuliert werden. Das heißt, daß bestimmte noch zu formulierende Filter Strukturen, die nicht parallel zu der Struktur des Matrixsatzes oder des Vorgängersatzes konstruiert sind, als nicht wohlgeformt ausfilterm. Das läßt den Schlu $B$ zu, da B bei den sogenannten kontextabhängigen Ellipsen in erster Linie LF-Filter, also grammatische Beschränkungen wirksam sind und dab für situative Ellipsen weniger grammatische als pragmatische Filter anzunehmen sind. Die Annahme von leeren Lexikoneinheiten ist in bezug auf Ellipsen mit sprachlichem Kontext noch vertretbar, da für jede antezedentielle Lexikoneinheit ein Pendant ohne PF in der unvollständigen Struktur angenommen werden kann. Schwierig dagegen dürfte die Auffassung zu vertreten sein, daß die D-Struktur von situativen Ellipsen mit leeren lexikalischen pro-Elementen bestückt ist. Nimmt man für syntaktische Strukturen von situativen Ellipsen lexikalische pros mit einer spezifischen Semantik an, könnte die Bestückung der Leerstellen mit lexikalischen pro-Elementen, die phonologisch repräsentierten Lexikoneinheiten entsprechen, zu unerwünschten semantischen Úbercharakterisierungen führen. Das würde der hier unterstützten Auffassung widersprechen, daß situative Ellipsen semantisch unspezifiziert sind.

Als Alternative zu der Annahme von leeren lexikalischen pro-Elementen ist der in 2.3. für situative Ellipsen verfolgte Weg anzusehen. Danach handelt es sich auch bei den D-Strukturen von kontextuellen Ellipsen um Strukturen, deren Endketten leere Kategorien aufweisen, die nicht durch lexikalische Einsetzung zustande kommen. Die leeren Kategorien sind phonologisch und semantisch leer Sie weisen einzig syntaktische 
Kat'(gorisierungsmerkmale und cinc syntaktische l'rojektionsstufe auf."I Durch semant jsche Interpretationsregeln mïßte dann die semantische Form ihres Antezedenten kopiert werden. Das setzt voraus, da B spätestens in der Logischen Form der Antezedent der leeren Kategorie jdentifiziert und durch Koindizierung gekennzeichnet ist. Die für die einzelnen Fälle noch zu formulierenden Kopierregeln setzen voraus, da.B es eine eine leere Kategorie enthaltende Struktur $A$ und eine andere Struktur B gibt. Die Regeln sind dadurch bedingt, da B A und B parallel strukturiert sind. Die eben genannte Alternative wird auch von WiLI.IAMs (1977) vertreten. Er läBt aber in kontextabhängigen elliptischen Strukturen nur leere Kategorien auf der untersten Projektionsstufe $\mathrm{X}^{\circ} \mathrm{zu}$.

Die Frage, ob für kontextabhängige Ellipsen $\mathrm{X}^{i}$ oder nur $\mathrm{X}^{0}$ zulässig ist, kann an dieser Stelle nicht beant wortet werden. Es soll auch nicht versucht werden, die einzelnen hier vorgestellten Varianten einzuschätzen. In bezug auf situative Ellipsen wurde das $Z$ iel verfolgt, eine Variante eingehend vorzustellen, un unter anderem eine Grundlage für die Entscheidung zu bieten, ob eine einheitliche Beschreibung von kontextucllen und situativen Ellipsen möglich ist.

In diesem Abschnitt sollte deutlich werden, daB situative Ellipsen sich von kontextabhängigen Ellipsen in LF darin unterscheiden, daB situative Ellipsen für ihre leeren Kategorien im Gegensatz zu kontextabhängigen Ellipsen keinen Antezedenten aufweisen und deshalb semantisch unspezifizierter sind.

\section{Die semantische Reprāsentation situativer Ellipsen}

Das zweite Kapitel ergab unter anderem, daB die Logische Form von situativen Ellipsen leere Kategorien enthält, die keinen Antezedenten aufweiseh. Für leere Kategorien in grammatisch determinierten Ellipsen, dio einen Antezedenten haben, kann man davon ausgehen, daB die semantische Form des Antezedenten durch bestimmte semantische Regeln irgendwie kopiert wird, so daB die SF der jeweiligen leeren Kategorie mit der semantischen Form des Antezedenten mehr oder weniger übereinstimmt. Bei situativen Ellipsen hingegen ist die Kopierung einer semantischen Form für eine leere Kategorie nicht möglich, da letztere keinen Antezedenten aufweist. Da aber für die semantigche Form eines Ausdrucks gilt, daß sie keine uninterpretierten leeren Kategorien enthalten darf, stellt sich die Frage, wie die leeren Kategorien ohne Antezedenten, die für situative Ellipsen typisch sind, interpretiert werden sollen. Die Beantwortung dieser Frage ist das hauptsächliche Anliegen dieses Kapitels. Bevor jedoch ein diesbezüglicher Vorschlag unterbreitet werden wird, soll das, was unter der semantischen Form zu verstehen ist; skizziert werden. Ich beziehe mich dabei auf Bierwisch (1983a, b, c, 1987) uni ZnMmenmann (1983, 1987).

21 Die Frage nach der Kasuszuweisung bei Nominalphrasen in kontextabhängigen Ellipsen wie (22) und (24) muß hier unbeant wortet bleiben. Ein Weg zu ihrer Beantwortung könnt $\theta$ sein, daß die Kasuszuweisung als ein Filter angesehen wird, der die Utbereinstimmung der Kasus von positionsgleichen Nominalphrasen $A$ und $B$ kontrolliert, wobei $A$ eine $\mathbf{N}^{2}$ der Struktur ist, die den Antezedenten enthält, und $B$ in der Struktur enthelten ist, die die leere Kategorie aufweist. 


\subsection{Theoretische Vorklärungen}

Eingangs sei darauf verwiesen, da $B$ die Darstellung der semantischen Form sprachlicher Ausdrïcke darauf zugeschnitten ist, die Voraussetzung zur semantischen Interpretation situativer Ellipsen zu schaffen. Damit soll gesagt sein, da B nur die für die Interpretation von situativen Ellipsen relevanten Aspekte der semantischen Form dargestellt werden, und daß ungelöste Probleme, die es in bezug auf die semantische Form reichlich gibt und die nicht im direkten Zusammenhang mit der semantischen Form von situativen Ellipsen stehen, in Kauf genommen werden müssen.

Das Repräsentationsformat von SF wird durch eine kategoriale Sprache festgelegt. Ihr Vokabular besteht aus Kategorien und Grundausdrücken. Die Grundausdrücke sind entweder Konstanten oder Variablen. Zum Bestand der Konstanten zählen die semantischen Primes wie zum Beispiel CAUS, TUN und INST, Konnektoren wie $\wedge, v, \rightarrow$, Negatoren und Operatoren wie zum Beispiel $v, x, \exists, \varepsilon$ und $\lambda$. Die Konstanten und Variablen und die aus ihnen bildbaren komplexen Ausdrücke sind kategorisiert. So ist mit anderen Worten jeder semantische Ausdruck ein Element einer Menge von semantischen Ausdrücken, die einer bestimmten Kategorie angehören. Es werden zwei Basiskategorien, $\mathrm{S}$ und $\mathrm{N}$, und die daraus bildbaren komplexen Kategorien des Typs $\left(\left(\alpha / \beta_{n}\right) / \ldots\right) / \beta_{1}$ unterschieden. Treffen semantische Ausdriicke der Kategorie $\left(\left(\alpha / \beta_{n}\right) / \ldots\right) / \beta_{1}$ auf semantische Ausdrücke des Typs $\beta_{1} \ldots \beta_{n}$, resultiert daraus ein semantischer Ausdruck der Kategorie $\alpha$. Fiir Lambdaabstraktoren gilt nach BIERwIScH (1987) folgende Eigenschaft:

(25) (a) Wenn $x$ eine Variable der Kategorie $\alpha$ ist, und Q ein Ausdruck der Kategorie $\beta$ ist, dann ist $\lambda_{1}\left[Q \mathbf{x}_{1}\right]$ ein Ausdruck der Kategorie $\beta / \alpha$.

(b) Wenn $\mathbf{P}$ ein Ausdruck der Kategorie $\beta / \alpha$ ist, $\mathrm{x}$ eine Variable der Kategorie $\alpha$ und $Z$ ein Ausdruck der Kategorie $\alpha$, dann gilt folgende Ãquivalenz

$\left[\lambda_{1}\left[\mathrm{Px}_{1}\right]\right] Z_{1}=\left[P Z_{1}\right]$

Die in (a) dargestellte Lambdaabstraktion kann auch wie folgt dargestellt werden:

(26) $A=\lambda_{1} \ldots \lambda_{n}\left[A x_{n} \ldots x_{1}\right]$

und $A \in M E\left(\left(\alpha / \beta_{n}\right) / \ldots\right) / \beta_{1}$

und $(1 \mathrm{i} \leq \leq \mathrm{n})^{22}$

In (25) (b) ist das Prinzip der Lambdakonversion dargestellt.

Von Brerwisch (1987, 96ff.) wird davon ausgegangen, daB in LF wie auf allen syntaktischen Ebenen lexikalische Einheiten (E) repräsentiert sind durch je ein Paar (PF, SF). PF ist dabei die phonologische Form von $\mathrm{E}$ und SF die semantische Form. Sowohl PF als auch SF von E sind im Lexikon, wo E dem Lexikoneintrag (LE) entspricht, fixiert. $\mathbf{E}$ erfüllt in LF die in LE angegebenen Kategorisierungs- und Subkategorisierungsbedingungen. Gemä $B$ jenen Bedingungen kann E in LF syntaktische Argumente haben. Alle Argumente von $\mathbf{E}$ werden in LF durch $\mathrm{E} \theta$-markiert, indem $\mathrm{E}$ ihnen eine $\theta$-Rolle zuweist. Die semantische Form von $\mathrm{E}$ enthält für jede Argumentstelle, die von E $\theta$-markiert werden kann, einen Abstraktor $\lambda_{1}$. BIERwISCH faBt somit $\boldsymbol{\theta}$-Rollen semantisch als Abstraktoren auf, die entsprechende Variablen in der SF von $\mathrm{E}$ binden. Die Abstraktoren sind bezüglich der jeweiligen dazugehörigen Argumentstellen indiziert. Im folgenden Beispiel geschieht das durch den regierten

$22 \mathrm{ME}$ ist die Abkürzung für 'meaningful expression'. 
Kasus 'akk' und das Merkmal '-reg', das das dazugehörige Argument als 'nicht von F. regiert' ausweist. Das folgende Beispiel demonstriert die $\boldsymbol{\theta}$-Markierung anhand eines vollständigen Satzes. Für das finite Verb ist unter seiner phonologischen Form seine (vereinfacht dargestellte) SF angegeben. 2 s

(27) (i) . . . [S $\left[\hat{\mathrm{y}}_{2} \text { Peter }\right]^{- \text {reg }}\left[\left[\left[\left[\left[_{\mathrm{x}^{2}} \text { ein Buch }\right]^{\text {akk }}\left[\mathrm{v}^{u}\right.\right.\right.\right.\right.$ nimmt $\left.\left.\left.\left.]\right]\right]\right]\right] \ldots$

$\lambda_{\text {akk }} \lambda_{-r e g} \lambda_{r}\left[\text { INST } x_{r}\left(\text { NEHM } x_{-r e g} x_{\text {akk }}\right)\right]^{24}$

Die folgenden semantischen Interpretationsregeln (28) und (29) verknïpfen die semantische form einer lexikalischen Einheit, die als Funktor kategorisiert ist, mit einem Argument.

(28) Wenn $\lambda_{1}$ eine $\theta$-Rolle einer lexikalischen Einheit ist nnd $\lambda_{1}$ die Argument position einer Konstituente $C$, die die SF-Repräsentation $Z$ hat, $\theta$-markiert, und die semantischen Kategorien von $Z$ und der von $\lambda_{i}$ gebundenen Variablen übereinstimmen, erhält $\lambda_{i}$ den Index der jeweiligen Argument position.

(29) Alle durch $\lambda_{1}$ gebundenen Variablen in SF übernehmen den Index $\mathrm{i}$.

Auf semantische Formen, die das Resultat der Anwendung von (29) sind, kann eine semantische Umformungsregel, die Lambdakonversion (25), angewendet werden. Das Wirken von (28) und (29) soll anhand des Beispiels (27) veranschaulicht werden. Die semantische Repräsentation des Tempus und des Satzmodus bleiben dabei unberücksichtigt. Mit SF' ist das Resultat der Anwendung der Lambdakonversion auf SF gemeint.

(27) (ii) SF : . . $\exists_{\mathrm{r}}\left[\right.$ peter $_{\mathrm{i}} \varepsilon_{\mathrm{k}}\left[\mathrm{BUCH} \mathrm{x}_{\mathrm{k}}\right] \lambda_{\mathrm{k}} \lambda_{\mathrm{i}}\left[\right.$ INST $\mathrm{x}_{\mathrm{r}}\left(\right.$ NEHM $\left.\left.\mathrm{x}_{\mathrm{j}} \mathrm{x}_{\mathrm{k}}\right)\right] \ldots$ (iii) $\mathrm{SF}^{\prime}: \ldots \exists_{\mathrm{r}}$ [INST $\left.\left(\mathrm{x}_{\mathrm{r}}\left(\mathrm{NEHM} \operatorname{peter}_{\mathbf{i}} \varepsilon_{\mathbf{k}}\left[\mathrm{BUCH} \mathrm{x}_{\mathbf{k}}\right]\right)\right)\right] \ldots$

\subsection{Die semantische Interpretation leerer Kategorien ohne Antezedenten}

Kommen wir auf das uns interessierende Problem zurück: Wie werden die im vorigen Kapitel repräsentierten Logischen Formen, die leere Kategorien ohne Antezedenten aufweisen, semantisch interpretiert? Sind die Kategorien INFL, ${ }^{1}$ und $\mathbf{V}^{\mathbf{i}}$ leer, kann die Regel (28) nicht auf die Strukturen, die sie enthalten, angewendet werden, denn es ist keine lexikalische Einheit vorhanden, die die jeweiligen syntaktischen Positionen $\boldsymbol{\theta}$-markieren könnte. Wie bereits an anderer Stelle bemerkt, können in vollständigen Strukturen nur die Subjektposition und die Komplementpositionen Argumentpositionen sein. All diese Positionen sollen deshalb auch in Strukturen mit leerer $\mathrm{V}^{i}$ und INFL' als Argumentpositionen angesehen werden. Es handelt sich dabei sozusagen um strukturell $\boldsymbol{\theta}$-markierte Argumentpositionen. Damit bei Abwesenheit eines $\boldsymbol{\theta}$-Markierers die semantischen Formen von Konstjtuenten, die sich auf einer Argumentposition befinden, als Argumente auf einen Funktor angewendet werden können, müssen die leeren Kategorien [vie] und $\left[_{1}, e\right]$ in SF als Funktorvariablen interpretiert werden. Für Funktorvariablen, die durch Großbuchstahen gekennzeichnet sind, wird die in (26) dargestellte Aquivalenzbeziehung zugrunde gelegt. Außer [vie] und $\left[{ }_{I}, e\right]$ sollen auch leere Kategorien, die

23 INST iat eine SF-Konstante der Kategorie (S/N)/S, die zu einem Sachverhaltstyp Instanzen bildet, ihm eine referentielle Lokalisierung gibt, die durch die Variable $\mathrm{x}_{\mathrm{r}}$ identifiziert wird. Die Variable $x_{r}$ wird bei unmarkiertem verbslen Modus existenzqusntifiziert. (Vgl. BIERWISCH (1987).)

${ }^{24}$ Die semantische Reprësentation ... [GEBEN $\mathbf{x}_{3} x_{2} x_{1}$ ] . . kann mit $x_{3}$ gibt $x_{2} x_{1}$ paraphrasiert werden. 
Argumentpositionen innehaben und keinen Antezedenten aufweisen, als Variable interpretiert werden.

Es wird davon ausgegangen, da $B$ die semantische Form eines komplexen Ausdrucks nur dann wohlgeformt ist, wenn die ihn konstituierenden Ausdrücke so kategorisiert sind, da $B$ auf sie die Lambdakonversion und gegebenenfalls andere hier nicht erwähnte semantische Operationen angewendet werden können. Diese Wohlgeformtheitsbedingung erfordert die Feststellung der passenden semantischen Form der Variablen, die die Interpretation von leeren Kategorien ohne Antezedenten sind.

Für die semantische Interpretation von leeren Kategorien als in einer bestimmten Weise kategorisierte Variablen schlage ich die Regeln (30) bis (32) vor.

(30) Eine leere Kategorie $X^{i}$ wird als Variable $x_{j}$ mit $x_{j} \in M E_{s}$ interpretiert, wenn (a) und (b) gelten.

(a) $\mathrm{X}^{\mathrm{i}}$ hat keinen Antezedenten

(b) $\mathbf{X}^{\mathbf{l}}$ kann alternativ zu e ein Subjekt und $\mathrm{X}^{\mathbf{i}-1}$ dominieren.

(31) Eine leere Kategorie $X^{i}$ wird als Variable $x_{1}$ mit $x_{1} \in M_{Y}$ interpretiert, wenn (a) und (b) gelten.

(a) $\mathrm{X}^{1}$ hat keinen Antezedenten

(b) $\mathrm{X}^{\mathrm{i}}$ kann alternativ zu e einen Determinator und $\mathrm{X}^{\mathrm{i}-1}$ dominieren. ${ }^{2} \mathrm{~J}$

(32) Eine leere Kategorie $X^{i}$ wird als Funktorvariable $\lambda_{1} \ldots \lambda_{n} \lambda_{r}$ [INST $x_{r}\left(\operatorname{Px}_{n} \ldots\right.$ $\left.\left.\mathbf{x}_{1}\right)\right]$ mit $\mathrm{P} \in \mathrm{ME}\left(\left(x / \beta_{\mathrm{n}}\right) \ldots\right) / \beta_{1}$ interpretiert, wobei $\beta_{1}$ der semant ischen $\mathrm{Kategorie}$ von $K^{i}$ gleicht, und $K^{i}$ das i-te semantische Argument von $P$ ist, wenn (a), (b) und (c) gelten.

(a) $\mathrm{X}^{1}$ hat keinen Antezedenten

(b) $\mathbf{K}$ ist die semantische Interpretation einer Konstituente oder einer leeren Kette, die eine Argumentposition $Y^{\mathbf{i}}$ besetzt.

(c) In LF gilt für $\mathrm{Y}^{i}$ :

$\ldots\left[_{\mathrm{INFL} 2} \mathrm{Y}^{\mathrm{n}}\left[\mathrm{INFL}^{0}\left\{_{\mathrm{V}^{2}}\left[\mathrm{~V}_{1} \mathrm{Y}^{\mathrm{n}-1} \ldots \mathrm{Y}^{1} \mathrm{~V}^{0}\right]\right]\right]\right] \ldots{ }^{06}$

Der Index i von $\mathrm{K}$ hzw. Y ergibt sich aus der syntaktischen Position der jeweiligen Argumente. Die Zählrichtung ist 'von V0 weg' festgelegt, da der jeweilige Funktor zuerst auf die semantische Interpretation des ihm in LF am nächsten stehenden Arguments angewendet wird.

Aufgrund der Regel (32) ist das jeweilige semantische Argument mit bestimmten Lambdaabstraktoren des Funktors koindiziert. Dadurch wird das gleiche Resultat wie bei der Anwendung der Regel (28) auf Strukturen mit lexikalisch realisierter Vo erzielt. Auf Strukturen, die das Ergebnis der Anwendung von (28) respektive von (30) bis (32) sind, kann die Lambdakonversion angewendet werden. Die Resultate dieser Umformung werden mit SF' bezeichnet.

Im folgenden sollen die hier eingeführten Regeln auf die Logische Form des Bei-

25 Unter Determinatoren werden hier abkürzend Artikel, die als $\mathbf{N}$ bildende Quantoren somantisch repräsentiert sind, verstanden.

26 Aus (32) (c) wird ersichtlich, daß diese Bedingung für Strukturen mit [Vie] und [INFLte] gilt, da nur deren semantische Form euf ein Argument angewendet werden kann, das außerhalb der strengen Subkategorisierungsdomäne steht. Fūr die semantische Interpretation von Nominalphrasen, attributiven Aljektivphrasen und Adverbphrasen mit leerem Kopf ergibt sich die Kategorie $\beta_{n}$ von $x_{n}$ auf eine andere Weise. Vergleiche dazu SchWABE (1987). 
spiels (33) angewendet werden. Mit $\mathrm{SF}^{\prime}$ ist eine L'mformung von SF gemeint. Der where Index $\mathrm{i}$ der Argumente $\mathrm{K}^{\mathrm{i}}$ in $\mathrm{SF}$ zeigt die aus (32) resultierende Reihenfolge der Argumente an, auf die die Funktorvariable $P$ angewendet wird. Unter den einzelnen Ausdriicken in $\mathrm{SF}$ ist jhre semantische Kategorisierung notiert.

(33) (i) $\mathrm{L} . \mathrm{F}:$ a) $\ldots \int_{\mathrm{INFL}_{2}}\left[\mathrm{~N}_{2} \mathrm{e}\right]^{3}\left[\mathrm{INFL}_{1} \mathrm{INFL}^{0}\left[\left[_{\mathrm{V}_{1}\left[\mathrm{~N}_{2} \mathrm{e}\right.}\right]^{2}\right.\right.$

[X: den Hammer $\left.\left.\left.]^{\prime}\left[\mathrm{V}_{0} \mathrm{e}\right] \mid\right]\right]\right] \ldots$

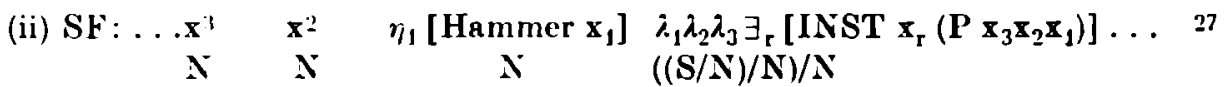

(iii) $\mathrm{SF}^{\prime}: \ldots \exists_{\mathrm{r}}\left[\mathrm{INST}{x_{\mathrm{r}}}_{\mathrm{P}}\left(\mathbf{P} \mathbf{x}_{\mathbf{3}} \mathrm{x}_{2} \eta_{1}\left[\mathrm{HAMMER} \mathbf{x}_{1}\right]\right)\right] \ldots$

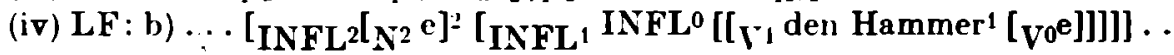

(v) SF : $x=\eta_{i}\left[\right.$ HAMMER $\left.x_{1}\right] \lambda_{1} \lambda_{2} \exists_{r}\left[\operatorname{INST} x_{r}\left(\operatorname{Px}_{2} x_{1}\right)\right] \ldots$

$$
N \quad N \quad(S / N) / N
$$

(vi) SF' : . . $\exists_{\mathrm{r}}\left[\right.$ INST $\mathrm{x}_{\mathrm{r}}\left(\mathrm{P} \mathrm{x}_{2} \eta_{1}\left[\right.\right.$ HAMMER $\left.\left.\left.\mathrm{x}_{1}\right]\right)\right] \ldots$

Fs wird deutlich, daß die semantischen Repräsentationen (33) (ii), (iii), (v) und (vi) freie Variablen enthalten. Diese werden, wie in Kapitel 4 zu sehen ist, in der konzeptu: ellen Struktur mit passenden Belegungen versehen.

\subsection{Die semantische Repräsentation des Satzmodus situativer Ellipsen}

Im vorausgegangenen Abschnitt wurden Logische Formen durch semantische Interpretationsregeln interpretiert. Für die syntaktischen Kategorien Spec und COMP0 wurden dabei keine semantischen Repräsentationen angegeben. Auch dieser Abschnitt kommt nicht zu einer abschließenden Lösung der Frage, inwieweit Spec und COMPO den Satzmodus determinieren. Sein Ziel besteht nur darin zu zeigen, daB für situative Ellipsen ein Satzmodus anzunehmen ist und wie dieser in SF zu repräsentieren ist.

Bereits in den Abschnitten 2.2. und 2.3. wurde dafür plädiert, situative Ellipsen wie die Beispiele (4), (5), (6), (10), (11), (12) als Sätze anzusehen. Argumentiert wurde unter anderem mit der auch auf situative Ellipsen zutreffenden Satzintonation, die in (34) den Fragemodus und in (35) den Deklarativ-oder Imperativmodus indiziert.

(34) /Eine Zigarette/

(35) /Den Hammer/

Weiterhin wurde das mögliche Vorkommen von Satzadverbialen wie in (36) und (37), von denen angenommen wird, daB sie den Grundmodus spezifizieren, als Indiz für die Satzartigkeit jener Ellipsen angesehen.

(36) Tatsächlich ein Mädchen!

(37) Vielleicht einen Schluck Wasser?

Es ist davon auszugehen, $\mathrm{da} B$ zu einer vollständigen semantischen Repräsentation eines Satzes die Repräsentation des propositionalen Gehalts PG und des Satzmodus

2i Der definite Artikel wird hier als kennzeichnender Operator $\eta_{1}$ repräsentiert. Auf Alternativen soll hier nicht weiter eingegangen werden. Desweiteren kann auch nicht diskutiert werden, wie in bezug auf die hier untersuchten Beispiele INFLo semantiach zu repräsentieren ist. 
ATT gehört. Der propositionale Gehalt identifiziert einen Sachverhalt und dessen temporale Einordnung. Der Satzmodus, ATT, bezeichnet die mit einem bestimmten propositionalen Gehalt verbundenen Einstellungen.

Für die semantische Repräsentation des Tempus soll folgendes festgelegt werden. 24 (38) $[+$ TEMPUS $] \rightarrow \lambda_{2} \lambda_{\mathrm{T}}\left[\mathrm{IN}\left(\mathrm{x}_{\mathrm{r}} \mathrm{T}_{1}\right) \wedge \mathbf{Q}\left(\mathrm{T}_{1} \mathrm{~T}_{0}\right) \wedge \mathrm{R}_{2} \mathbf{x}_{\mathrm{r}}\right.$

Das syntaktiache Merkmal (TFMPCS), das in dem hier vert retenen syntaktischen Rahmen (9) von INFLo dominiert wird, wird als zweistelliger Funktor interpretiert. Unter $T_{1}$ ist der Zeitabschnitt zu verstehen, $z u$ dem der die Variable $\mathbf{x}_{r}$ spezifizierende Sachverhalt besteht, der durch $\mathbf{P}$ charakterisiert ist. $T_{0}$ ist die Zeitspanne, in der die Sprechhandlung stattfindet. Die Variable $Q$ ist als eine Variable über bestimmte semantische Konstanten anzusehen. Sie wird in Abhängigkeit von der Tempusspezifizierung des jeweiligen Ausdrucks durch die Konstanten NACH, VOR oder IN besetzt. Ist der Satz bezüglich des Tempus nicht spezifiziert, bleibt $\mathbf{Q}$ als Variable erhalten, wenn nicht, wie unten ausgefiihrt, die Spezifizierung von $Q$ in Abhängigkeit von ATT erfolgt. Die Variable $R_{2}$ wird durch $\lambda_{T}$ [INST $x_{r}$ P] ersetzt.

Nachdem in aller Kürze einige Annahmen zur Repräsentation des Tempus vorgestellt worden sind, ist zu der vollständigen semantischen Repräsentation des Satzes die Repräsentation des Satzmodus hinzuzufügen. Dabei gehen wir davon aus, da B der Satz in der Regel iiber bestimmte Ausdrucksmittel verfügt, die den Satzmodus repräsentieren. Die Frage, wie der Satzmodus zu repräsentieren ist, und welche sprachlichen Mittel ihn ausdrücken, kann aufgrund der gegenwärtigen Forschungslage noch nicht umfassend beant wortet werden.

Die Fxistenz des Satzmodus ist damit zu begrïnden, da B mit einem Satz nicht nur die sprachlich kodierte Sachverhaltsrepräsentation PG gegeben ist, sondern auch die Vorschrift, wie dieser Satz kognitiv und kommunikativ zu verarbeiten ist.?" Die eben genannte Vorschrift ist als der Inhalt des Satzmodus anzusehen. Nach I,ANG ist ein Sachverhalt $p$ als eine Konfiguration von Konzepten aufzufassen, die aus der Interaktion verschiedener Subsysteme des Systems der konzeptuellen Strukturbildung (S resultiert. Die dem Sachverhalt p entsprechende Konfiguration von Konzepten wird durch eine Einstellung oder durch eine Konfiguration von Finstellungen bewertet. Die Einstellungen werden, so LANG, durch das System $C_{A}$ determiniert. Es werden in der Hauptsache epistemische, emotionale und intentionale Einstellungen unterschieden. Die sprachliche Repräsentation der Einstellungen, die einen Sachverhalt bewerten, ist der Satzmodus. Die semantische Repräsentation des Satzmodus erfolgt durch die Metavariable ATT. ATT ist als ein komplexer Funktor anzusehen, dessen eine Variable durch PG und dessen andere durch den Einstellungsträger spezifiziert wird. Es ist davon auszugehen, da B ATT das Ergebnis der Interpretation bestimmter terminaler Bestandteile der Jogischen Form eines Satzes ist. Zu den Determinanten von A'TT wird der Verbmodus, werden Modalverben, Satzadverbien und bestimmte Intonationsmuster gezählt.

Wie bereits erwähnt, ist ATT eine Metavariable für die Repräsentation des Satzmodus in der semantischen Form des Satzes. Sie kann durch bestimmte Grundnodi belegt werden, wobei davon auszugehen ist, daß ein Satz nur einen Grundmodus haben

28 Die semantische Reprësentation ales Tempus, die aus methodologischen Gründen un dieser Stelle erfolgt, verdanke ich den Hinweigen von Zimmermans.

29 Bei meinen Darlegungen zum Satzmodus beziehe ich mich auf Ausführungen von LANG, die in Rahmen der Vorbereitung auf das Projekt 'Nitzmodus' prfolgten. 
kamn. Der (irundmodus kamn durch die semantische Form weiterer modaler Konstituenten spezifiziert oder modifiziert werden. Der (inundmodus resultiert, so die vorläufige Auffassung, aus morphosyntaktischen Kriterien in bestimmter Kombination mit 'Intonationsmustern'. Fs werden vorerst drei Grundmodi unterschieden: der 'Interrogativ', der 'Deklarativ' und der 'Imperativ', dariiber hinaus wird ein weiterer (irundmodus, der 'Exklamativ', erwogen. ATT kann demzufolge alternativ mit der kemantischen Repräsentation dieser drei Grundmodi belegt werden. Es sollen nun die drei möglichen Belegungen für ATT kurz dargestellt werden. Diese Darstellungen können im Rahmen dieser Arbeit nicht weiter diskutiert werden.

(39) Deklarativ: DECL

Interrogativ:

INT

Imperativ :

IMP30

Die hier in aller Kürze vorgenommene Darstellung des Satzmodus soll mit der Nellnung einiger vor allem für diesen Artikel relevanten Fragen abgeschlossen werden.

1. Woraus resultiert die semantische Repräsentation des Satzmodus, mit anderen Worten, wodurch ist der Grundmodus determiniert und wodurch wird er modifiziert oder spezifiziert? In wieweit trägt die semantische Interpretation der von Spec bzw. COMPo dominierten Einheiten zur Ermittlung von ATT bei? Welche Rolle spielen die Satzadverbiale in bezug auf ATT? Gibt es bestimmte Selektionsbeschränkungen bezüglich ATT und PG?

2. Wie ist die Annahme von drei, eventuell von vier Grundmodi zu rechtfertigen? Wie und wodurch können sie modifiziert und spezifjziert werden? Welche Untertypen sind in bezug auf die Grundmodi anzunehmen?

3. Haben elliptische Sätze einen Satzmodus? Wenn ja, schließt sich die Frage nach dessen Repräsentation an. Was ist, wenn die Frage mit nein beantwortet wird? Letztere Fragen betreffen vor allem Ellipsen, bei denen Spec bzw. COMPN in LF nicht das Merkmal [MODUS] und eine w-Wort-Phrase dominiert. Bei diesen Ellipsen könnte die Intonation als einziger Indikator für einen bestimmten Modus angesehen werden.

J)as, was eben in einer kurzen Skizze zum Satzmodus gesagt wurde, ist erst in einigen Ansätzen erforscht worden, so daB die eben gestellten Fragen im Rahmen dieser Arbeit offen bleiben. Die Rechtfertigung obiger Skizze sowie die Beantwortung der oben gestellten und vieler anderer Fragen ist ein Forschungsprogramm, das in den kommenden Jahren eine Gruppe von Wissenschaftlern beschäftigen wird. Aufgrund der gegenwärtigen Forschungslage kann hier kaum etwas zum Satzmodus von Ellipsen ausgesagt werden. Gleiches gilt auch für die Interpretation von Spec b̌w. COMPo, die, so kann angenommen werden, zu den Determinanten des Satzmodus gehören. So befinden wir uns nun in dem Dilemna, daß einerseits nur Provisorisches zum Satzmodus von Ellipsen gesagt werden kann, andererseits aber etwas dazı festgelegt werden muß. Die Notwendigkeit einer vorläufigen Festlegung des Satzmodus für Ellipsen ergibt sich aus der Zielstellung dieses Aufsatzes, nämlich unter anderem die Außerungsbedeutung und den kommunikativen Sinn von Ellipsen zu ermitteln.

Eine Voraussetzung der Bestimmung der $\AA_{u}$ Berungsbedeutung und des kommunikativen Sinns ist der Satzmodus, da dieser zum einen die Erfüllungsbedingungen für den

:0 $\mathrm{Vgl}$. Мотsch/Pasch (1987). 
in PG kodierten Sachverhalt in bezug auf den ĀuBerungskontext determiniert und zum anderen den kommunikativen Sinn, mit dem der Satz als Āu Berung in den Interaktionskontext eingeführt wird, bestimmt ${ }^{31}$. Aufgrund der Forschungslage und der Zielstellung dieses Artikels müssen bezüglich ATT von elliptischen Sätzen bestimmte provisorische Festlegungen vorgenommen werden: Weisen situative Ellipsen ohne finites Verb einen steigenden Intonationsverlauf auf und/oder eine w-Phrase, kann für sie der Interrogativmodus angenommen werden, da w-Phrasen und steigende Intonation als Indikatoren für den Interrogativ gelten. ${ }^{32}$ Ist der Intonationsverlauf fallend, und sind keine weiteren Indikatoren, für ATT vorhanden, ist ATT entweder durch IMP oder durch DECL zu ersetzen, denn sowohl der Deklarativsatz als auch der Imperativsatz besitzen einen fallenden Intonationsverlauf. Das bedeutet, da $B$ eine situative Ellipse, weist sie als einzigen Indikator für ATT einen fallenden Intonationsverlauf auf, in bezug auf ATT mehrdeutig ist.

Zur semantischen Interpretation des Tempus und des Modus situativer Ellipsen sollen nun zwei Beispiele folgen. Die syntaktischen Informationen, denen ein bestimnter Intonationsverlauf entspricht, sind in LF unter COMP ${ }^{0}$ notiert. $3: 3$

(40)/Eine Zigarette/

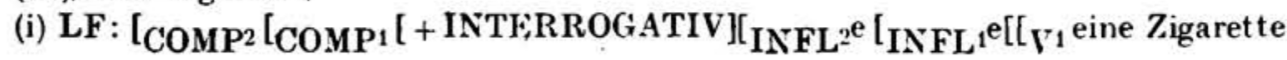

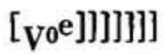

(ii) SF: INT $\left[\exists_{r}\left[\operatorname{IN}\left(x_{r} T_{1}\right) \wedge Q\left(T_{1} T_{0}\right) \wedge \operatorname{INST}\left(x_{r}\left(P_{\varepsilon_{1}}\left[\right.\right.\right.\right.\right.$ ZIGARETTE $\left.\left.\left.\left.x_{1}\right] x_{2}\right)\right]\right]$

Das Merkmal [ + INTERROGATIV] unter COMP0 in (40)(i) ist als Eingabe für die phonologische und die semantische Interpretation anzusehen. Da B es hier vorkommt, ist damit zu begründen, da B / Eine Zigurette/ eine Frageintonation aufweist, die als Indiz für die Realisierung des Satzmodus dieser Ellipse angesehen werden kann. Das Vorkommen dieses Merkmals kann dann erst funiert begriindet werden, wenn die S-Struktur und die sie bildenden Regeln jener elliptischen Ausdrücke eingehender untersucht sind. Das konnte im Rahmen dieses Aufsatzes leider nicht bewerkstelligt werden, so da $B$ wir uns mit der provisorischen Festlegung dieses Merkmals begnügen müssen. Da keine Indikatoren für das Tempus in (40) gegeben sind, wird es durch eine entsprechende Variable $(Q)$ repräsentiert.

(41) /Den Hammer/

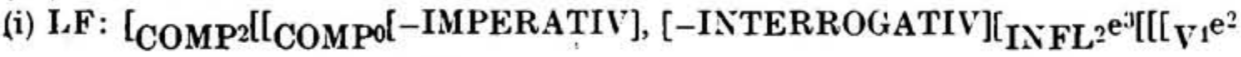

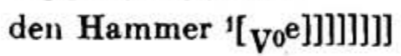

(ii) $\mathrm{SF}^{\prime}: \operatorname{DECL}\left(\exists_{\mathrm{r}}\left[\mathrm{IN}\left(\mathrm{x}_{\mathrm{r}} \mathrm{T}_{1}\right) \wedge \mathrm{Q}\left(\mathrm{T}_{1} \mathrm{~T}_{0}\right) \wedge \operatorname{INST} \mathrm{x}_{\mathrm{r}}\left(\mathrm{Px}_{3} \mathrm{x}_{2} \eta_{1}\left[\mathrm{HAMMER} \mathrm{x}_{1}\right]\right)\right]\right]$

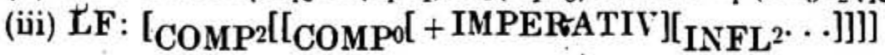

(iv) $S_{\mathrm{F}^{\prime}}: \operatorname{IMP}\left(\exists_{\mathrm{r}}\left[\mathrm{IN}\left(\mathrm{x}_{\mathrm{r}} \mathrm{T}_{1}\right) \wedge \mathrm{NACH}\left(\mathrm{T}_{1} \mathrm{~T}_{0}\right) \wedge \operatorname{INST} \mathrm{x}_{\mathrm{r}}\left(\mathrm{P}\right.\right.\right.$ hörer $\mathrm{x}_{2} \eta_{1}\left[\right.$ HAMMER $\left.\left.\left.\left.\mathrm{x}_{1}\right]\right)\right]\right]$ Der Satzmodus wird in (41) aufgrund der oben erwähnten Mehrdeutigkeit in (ii) als DECL und in (iv) als IMP repräsentiert. ${ }^{34}$ Im Skopus von IMP wird Q durch $\mathrm{NACH}$

31 Siehe Motsch/PASch (1987) und LANg/Pasch (1985).

32 Vgl. Heidolph (1981, 8i5 ff.).

${ }^{33} \mathrm{Da}$ [ [ + INTERROGATIV] in (33) von COMP0 dominiert ist, gehört, wie bereits erwähnt, zu den Provisorien dieses Aufsatzes. Ebenfalls müBte durch weitere Untersuchungen überprüft werden, ob $x_{r}$ bei Entscheidungsfragen existenzquantifiziert ist.

${ }^{34} \mathrm{Ob}$ (ii) eine wohlgeformte kommunikative und konzeptuelle Interpretation crfaliren kann, wird in SCHWABE (1987) diskutiert. 
spezifiziert, da eine andere Belegung nicht möglich ist. Weiterhin wird im Skopus von I.MP die Variable $x_{3}$ mit hörer helegt, da bei Imperativen das Subjekt immer den Hörer bezeichnet. 35

Daß die syntaktischen Repräsentationen der in diesem Aufsatz analysierten situativen Ellipsen leere Kategorien aufweisen, und da $B$ diese als freie Variablen in SF repräsentiert sind, trägt dem hier verfolgten methodischen Grundsatz (7) Rechnung, daB in einer sprachlichen Struktur nicht mehr repräsentiert sein darf, als wirklich sprachlich ausgedrïckt ist. So enthalten die hier angegebenen semantischen Repräsentationen nicht mehr an Informationen als syntaktisch indiziert ist. Wie im folgenden Kapitel gezeigt werden wird, werden die semantischen freien Variablen durch Entitäten des AuBerungskontextes beziehungsweise des Interaktionskontextes belegt.

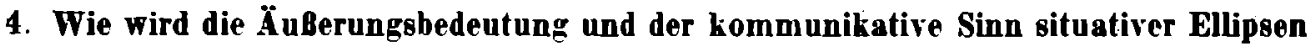 ermittelt?}

\subsection{Theoretischer Hintergrund}

In vorangegangenen Kapitel wurden die semantischen Formen der syntaktisch und semantisch mehrdeutigen situativen Ellipse Den Hammer dargestellt. Unter situativen Ellipsen wurden unvollständige Ausdrücke verstanden, in deren Logischer Form leere Kategorien ohne Antezedenten vorkommen und in deren semantischer Form diese in LF nicht koindizierten leeren Kategorien als freie Variablen interpretiert sind.

In diesem Kapitel soll nun geklärt werden, wie diese Variablen weiter interpretiert werden. Es wird dabei von der Annahme ausgegangen, daß sie aufgrund bestimmter IRegeln und unter Einhaltung bestimmter Bedingungen durch Einheiten des situativen und kommunikativen Kontextes belegt werden. Da diese sowohl durch das konzeptuelle als auch das kommunikative Kenntnissystem determiniert sind, werden in diesem Kapitel die Außerungsbedeutung und der kommunikative Sinn im Zusammenhang behandelt. Zunächst aber wird versucht, die Begriffe 'Āußerungsbedeutung' und 'kommunikativer Sinn' in einer für den Bedarf dieses Aufsatzes angemessenen Weise zu erklären. Auf diese notwendigen theoretischen Vorklärungen folgt ein Vorschlag, für (41) die Außerungshedeutung und den kommunikatjven Sinn zu ermitteln.

Die Darstellung der Außerungsbedeutung stützt sich auf Ideen von BLEBwisch (1983 a, 1983 b), LANG (1983) und Motsch/Pasch (1987). Es ist davon auszugehen, daB sich die Außerungsbedeutung komplexer semantischer Formen aus der schrittweisen Anwendung der Interpretationsfunktion g auf die einzelnen semantischen Formen, die die komplexe semantische Form konstitujeren, und auf einen möglichen Kontext ergibt.30

(42) $\mathrm{g}\left(\mathrm{SF} \mathrm{ct}_{\mathrm{CS} / \mathrm{CA}}\right)=\mathrm{m}$

Unter einem 'möglichen Kontext' wird der Kontext verstanden, der bestimmte Bedingungen, die durch die jeweilige SF fixiert werden, erfüllt. Von BIERwisch (1983a) wird der Kontext als ein mentales Modell beschrieben, das als Repräsentation der

45 Aufgrund der Alternativen ein oder zwei Komplemente und Imperativ- oder Deklarativestz, hat das Beispiel vier syntaktische und semantische Reprägentationen. Vgl. (19)(a) und (b).

अi Vgl. BLERTSCH $(1983 \mathrm{c}, \mathbf{7 8})$. 
Kenntnisse iiber eine mögliche oder eine aktuelle Situation aufzufassen ist. Die (iesamtheit der mentalen Modelle wird durch das konzeptuelle System CS determiniert. Durch ATT und das Tempus wird in der semantischen Form eines Satzes bestimmt, ob es sich bei dem Kontext um eine mögliche oder aktuelle Situation handeln muB. Die in (42) angegebene Funktion setzt voraus, daB die konzeptuellen Einheiten (m) wie die semantische Form (SF) einem bestimmten Typ angehören. Der Typ der konzeptuellen Einheit muß dabei mit der Kategorie der semantischen Form korrespondieren, auf die die Funktion g angewendet wird. ${ }^{37}$

Zu den hier vorgenommenen Repräsentationen der Āußerungsbedeutung von situativen Ellipsen seien einige Bemerkungen vorausgeschickt. Erstens, es handelt sich nicht wie bei den Repräsentationen syntaktischer Strukturen und der semantischen Form um formale Repräsentationen. Die zweite Bemerkung gilt der konzeptuellen Interpretation des Einstellungsoperators ATT. Einer befriedigenden konzeptuellen Interpretation der Belegung von ATT stehen hauptsächlich zwei Tatsachen entgegen. Zum einen steckt die Forschung zur semantischen Repräsentation des Modus - wie bereits erwähnt - noch in den Anfängen, zum anderen ist bislang über die Repräsentation von Einstellungen in CA kaum etwas bekannt. So ist es nicht ausgemacht, daß Einstellungen in CA als Operatoren wie in SF repräsentierbar sind. (Gegen diese Annahme spricht, da B Einstellungen in SF auch propositional repräsentiert sein können.:IK Aufgrund dieser Unklarheiten soll im Rahmen dieses Aufsatzes für ATT[P(i] als ĀuBerungsbedeutung angenommen werden: Der Produzent der Äußerung (Sprecher) gibt $z u$ verstehen, duß er eine Einstellung(e) zu einem Sachverhalt (sv) hat. Die chen paraphrasierte Āußerungsbedeutung soll durch e(sv) zusammengefaßt werden. Die Einstellung (e) ist dabei unter Berücksichtigung der oben genannten Vorbehalte als die kontextuelle Interpretation des Einstellungsoperators ATT zu verstehen. Dem propositionalen (iehalt (P(i) entspricht auf der konzeptuellen Ehene ein Sachverhalt (sv). Die aufgrund des Beispiels (41) uns interessierenden Modi 'Deklarativ' und 'Imperativ' haben in Anlehnung an Motscil/Pasch (1987) die folgende Außerungsbedeutung:

(43) (i) $g\left(\right.$ JECL[P(i], ct $\left(\mathrm{s} / \mathrm{cA}_{A}\right)$ : Sprecher gibt zu erkennen, daß er von sv iiberzeugt ist.

(ii) $g\left(I M P\left[P(i], t_{\left.\operatorname{cs} / c_{A}\right)}\right)\right.$ : Sprecher gibt zu erkemnen, daß er will, daß Hörer sv realisiert.

Mit Motsch/Pasch (1987) wird hier davon ausgegangen, da B sich der kommunikative Sinn einer Außerung aus der Außerungsbedeutung e(sv), der ihr zugewiesenen illokutiven Funktion IF und der Anwendung von e(sv) und IF auf den Interaktionskontext ct $_{\text {I }}$ ergibt. Illokutive Funktionen sind durch eine mit der Āußerung verbundenen Zielhierarchie mit einem fundamentalen Ziel, durch eine Menge von konstitutiven Bedingungen und durch konventionelle soziale Konserquenzen bestimmt.19 Nach Motsch/Pasci ist die illokutive Grundfunktion als das Resultat der Anwendung von bestimmten Regeln auf den Einstellungsoperator ATT anzusehen. Jene Regeln gehören zum Inventar des kommunikativen Kenntnissystems. In dem folgenden Schema sind die illokutiven Funktionen, die DECL und JMP zugeordnet sind, aufgeführt. Die illokutiven Funktionen werden durch das fundamentale illokutive Ziel, das mit der jeweiligen Außerung verbunden ist, repräsentiert.

37 Vgl. LANG (1983).

j8 Vgl. Motsch/Pasch (1987).

39 Ebcla. 
(44) (i) DFCL[P(i]: Hörer glaubt sv

(ii) IMP [P(i]: Hörer führt sv aus

Wie nun die ĀuBerungsbedeutung und der kommunikative Sinn von (41) zustande kommen, soll im folgenden Abschnitt dargestellt werden.

\subsection{Ein Beispiel für die Repräsentation der Außerungsbedeutung und des kommuni- kativen Sinns situativer Ellipsen}

Die Außerungshedeutung und der kommunikative Sinn sind im Rahmen der hier vertretenen Theorie verbunden mit dem Erfültsein bestimmter Bedingungen, die durch die semantische Form, durch die illokutive Funktion und durch bestimmte kommunikative Prinzipien determiniert sind.10 Die durch die semantische Form fixierten Bedingungen werden oder werden nicht durch den Kontext $\mathrm{ct}_{\mathrm{cs} / \mathrm{CA}}$ erfüllt. Der Interaktionskontext ct ${ }_{\text {I }}$ erfüllt oder erfüllt nicht die durch die illokutive Funktion und durch bestimmte kommunikative Prinzipien gegebenen Bedingungen. Ist wie bei dem Beispiel (41) die semantische Form weitgehend unspezifiziert, so ist die Außerungsbedeutung mehr auBersprachlich, durch den Kontext, determiniert.

Die Ermittlung der Außerungsbedeutung und des kommunikativen Sinns soll anhand der semantischen Form (41) (iv) dargestellt werden.

(41) (iv) IMP $\left[\exists_{r}\left[\operatorname{IN}\left(x_{r} T_{1}\right) \wedge N A C H\left(T_{1} T_{0}\right) \wedge \operatorname{INST} x_{r}\left(P\right.\right.\right.$ hörer $x_{2} \eta_{1}\left[\right.$ HAMMER $\left.\left.\left.\left.x_{1}\right]\right)\right]\right]$

Es wurde davon ausgegangen, da $B$ sich die Außerungsbedeutung komplexer semantischer Formen wie (41) (iv) aus der schrittweisen Anwendung der Interpretationsfunktion g auf die die komplexe semantische Form konstituierenden semantischen Formen und auf den Kontext ergibt. Dadurch wird der jeweiligen semantischen Form eine konzeptuelle Einheit eines entsprechenden Typs zugewiesen. Die semantische Form (41) (iv) soll auf den folgenden Kontext bezogen werden.

(45) Der Sprecher möchte ein Bild an die Wand nageln. Ihm fehlt dazu ein Hammer.

Dieser liegt auf dem Tisch. Unweit des Sprechers steht der Hörer.

Wie leicht zu erkennen ist, enthält die $S F^{\prime}(41)$ (iv) zwei freie Variablen $P$ und $x_{2}$. Die semantische Form $\eta_{1}$ [HAMMER $\mathrm{x}_{1}$ ] fixiert die Bedingung, da $B$ der Referent von $x_{1}$ ein bestimmtes Individuum aus der Menge der Individuen sein mu $B$, die die Eigenschaft haben, ein Hammer zu sein. Für $\eta_{1}$ [HAMMER $x_{1}$ ] soll als Au Berungsbedeutung 'ein bestimmter Hammer' angenommen werden. Im Gegensatz zu $x_{1}$ ist die Referenz von $x_{2}$ und $P$ sprachlich nicht festgelegt. Sprachlich determiniert ist nur, da $B$ es sich bei $x_{2}$ um ein Argument eines dreistelligen Funktors und bei $P$ um den Funktor selbst handelt, von dem das erste Argument $\eta_{1}$ [HAMMER $x_{1}$ ], das zweite $x_{2}$ und das dritte Argument hörer ist. Wird, wie in (46) unten veranschaulicht, die Interpretationsfunktion $g$ auf eine freie Variable, deren möglicher Referent durch nichts als durch einen bestimmten Typ, dem er angehören muB, eingeschränkt ist, und den Kontext ct cs/CA $_{\text {A }}$ angewendet, sucht $\mathrm{g}$ für die jeweilige Variable eine Einheit $\mathrm{m}$ in ct $_{\mathrm{Cs}}$.

(46) $g\left(x_{1}\right.$ ct $\left.\mathrm{Cs} / \mathrm{CA}_{\mathrm{A}}\right)=\mathrm{m}$

Die Belegung der jeweiligen Variable mit $\mathrm{m}$ ist dabei nicht willkürlich. Sie hängt von bestimmten Bedingungen ab. Die erste Bedingung, die aktuelle Kontextbedingung, beinhaltet, da $B$ bei elliptischen AuBerungen ohne Tempusindizierung, ohne deiktische Ausdrücke und ohne sprachlichen Kontext die eemantische Form auf den aktu-

$40 \mathrm{Zu}$ diesen kommunikativen Prinzipien gehört beispielsweise das Kooperationsprinzip von Grice (1968). 
ellen Kontext zu beziehen ist. Die eben genannte Bedingung ist mit der in Motsch/ Pascri (1987) erwähnten Verstehensbedingung zu begründen: Damit der Sprecher das mit seiner Āußerung verbundene fundamentale Ziel erreicht, nuB der Hörer verstehen, was der Sprecher von ihm will. Das Verstehen ist aber nur dann gesichert, wenn der Sprecher und der Hörer die AuBerung phonologisch, syntaktisch und semant isch gleichermaßen strukturieren, wenn sie sie auf den gleichen Außerungskontext und auf den gleichen Interaktionskontext beziehen. Ist der Kontext $\mathrm{ct}_{\mathrm{cs} / \mathrm{CA}}$ nicht durch einen Vorgängertext und/oder nicht durch die grammatisch determinierte Bedeutung festgelegt, wird die semantische Form der ĀuBerung auf den aktuellen Kontext bezogen, da dieser mit der Āußerung immer gegeben ist und somit auch dem Hörer im Bewußtsein präsent ist. Die Bedingung, da B der Sprecher und der Hörer die semant ische Form auf den gleichen Kontext beziehen, ist damit erfüllt. Da die Variablen durch konzeptuelle Einheiten belegt werden, die durch den aktuellen Kontext gegeben sind, kann von einer spezifischen Belegung gesprochen werden. ${ }^{4 l}$ Eine zweite, mit der aktuellen Kontext bedingung eng verknüpfte Bedingung ist, da B der Sprecher und der Hörer die semantischen Variablen mit den gleichen konzeptuellen Einheiten belegen. So kann in bezug auf das Beispiel (33) (v) bzw. (iv) die Variable $\mathbf{P}$ nicht die ĀuBerungsbedeutung 'reparieren' haben, wenn der Hörer nicht weiß, daB der Hammer entzwei ist. Oder, die Variable $x_{2}$ in (41) (iv) kann in dem unter (45) angegebenen Kontext nicht mit 'Fritz' belegt werden, da 'Fritz' nicht zu dem bewuBten Kontext gehört und somit nicht im Bewußtsein des Hörers präsent ist. Anders gesagt, die vom Sprecher vorgesehene Variablenbelegung darf nicht für den Hörer eine neue Information darstellen. Sie mu B schon vor der Außerung im internen Modell des Hörers vorhanden oder für ihn erschließbar sein. Da die entsprechenden Variablen zum Topik des Satzes gehören, soll die Bedingung Topikbedingung genannt werden. ${ }^{42}$ In engem Zusammenhang damit zu sehen ist die Bedingung, daß der Sachverhalt mit den Einstellungen, die mit ihm verknüpft sind, vereinbar sein muB. Er muB so beschaffen sein, da B der Sprecher vorgeben kann, von ihm überzeugt zu sein, daß der Sprecher ihn erfragen kann oder da $B$ er den Hörer auffordern kann, ihn zu realisieren. Diese Bedingung soll als Einstellungsbedingung bezeichnet werden. So kann beispielsweise $P$ in (41) (iv) nicht mit 'geben' belegt werden, wenn der Kontext so beschaffen ist, daß der Sprecher den Hammer bereits hat. Die eben genannten, ziemlich allgemein gehaltenen Bedingungen gelten für die Variablenbelegung. Sie sollen deshalb auch Belegungsbedingungen genannt werden. Sie gehören zu den Verwendungsbedingungen von situativen Ellipsen. Au Ber ihnen gibt es weitere Verwendungsbedingungen. Eine, die Redundanzbedingung, beinhaltet, daß die Åußerung unter bestimmten Umständen weitgehend redundanzfrei sein sollte. Die Forderung der Einsparung von redundanten Einheiten ergibt sich zum Beispiel daraus, daß Redezeit, Papier und im Falle von Anzeigen und Telegrammen Geld gespart werden soll. AuBerungen, die weitgehend redundanzfrei sind, sind außerdem für die Hörer schneller und besser zu erfassen. Als Beispiele für Kontexte, in denen es dem Sprecher auf die eben genannten Einsparungen ankommt, sind (i), (ii) und (iii) anzusehen:

(i) Ein Unfall hat sich ereignet. Auf der Straße liegt eine bewuBtlose Person.

\section{A : Einen Krankenwagen!}

41 Vgl. SHOPEN (1972 und 1973), der die spezifische, nicht variierbare Belegung als 'definite ellipsis' bezeichnet.

42 Vgl. Pasch $(1983,104)$ und Pasch/Zimmermanin (1983). 
(ii) Im Operationssaal. Nur das Notwendigste darf gesprochen werden.

A : Tupfer!

(iii) Ein Schild an einem Schaufenster: Heute lebendes Fischfutter!

SchlieBlich sei eine weniger allgemeine Bedingung genannt. Sie betrifft die sozialen Beziehungen zwischen dem Sprecher und dem Hörer. Gemeint sind danit bestimmte Erfordernisse an die Form der AuBerung. Weiß der Sprecher, daB der Adressat in der suzialen Hierarchie über ihm steht, und er mit dem Hörer weder verwandt noch befreundet ist, so darf er dem Adressaten gegenüber keine situative Ellipse verwenden. Ein Angestellt er kann seinen Direktor beispielsweise nicht beim Essen mit der AuBerung (47) Das Salz bitte!

um Salz bitten. Diese die besondere soziale Konstellation von Sprecher und Hörer betreffende Bedingung soll Vèrtrautheitsbedingung genannt werden. ${ }^{\text {is }}$

AuBer den Belegungs- und Kooperationsbedingungen zählen zu den Verwendungsbedingungen von situativen Ellipsen sogenannte Erfülltseinsbedingungen. Damit sind die durch die semantische Form gegebenen Bedingungen und ihr Erfülltsein durch den Kontext ct ${ }$ cs gemeint. Diese Bedingungen gelten für alle sprachlichen AuBerungen und sind im Gegensatz zu den Belegungsbedingungen nicht typisch für situative Ellipsen.

Zu den hier aufgezählten Verwendungsbedingungen von situativen Ellipsen sei abschließend hinzugefügt, daB sie sicherlich nicht alle Verwendungsbedingungen ausmachen. Eine umfassendere Darstellung von allgemeinen und speziellen den $\mathrm{AuBe}-$ rungsty $p$ betreffenden Verwendungsbedingungen steht somit noch aus. Diese Darstellung müßte neben der Aufzählung der relevanten Bedingungen auch das Verhältnis der Bedingungen zueinander enthalten.

Nachdem die Regel zur Belegung von freien Variablen in der semantischen Form von situativen Ellipsen und die dabei geltenden Bedingungen sowie der damit verbundene theoretische Rahmen angegeben worden sind, können die Ảußerungsbedeutung und der kommunikative Sinn des Beiepiels (41) (iv) ermittelt werden.

(48) $\mathbf{g}\left((41)(i v)\right.$, $\left.\mathrm{ct}_{\mathrm{CS} / \mathrm{CA}_{\mathrm{A}}}\right)$ : Der Sprecher gibt zu erkennen, daB der Sprecher wünscht, da B der Hörer realisiert, daB der Hörer dem Sprecher den Hammer gibt.

Die aktuelle Kontextbedingung erscheint bei diesem Beispiel insofern als irrelevant, als daß durch den Operator IMP in SF gefordert wird, da B in seinem Skopus das Tempus so repräsentiert ist, da $\beta$ der durch PG bezeichnete Sach verhalt nach dem Außerungszeitpunkt liegt. Die aktuelle Kontextbedíngung kommt aber ins Spiel, wenn der Referent von $x_{2}$, identifiziert werden soll. So wird $x_{2}$ durch $g$ mit 'Sprecher' belegt, da dieser zum aktuellen Kontext des Sprechers und des Hörers gehört. Damit ist auch die Topikbedingung berücksichtigt, die auch in bezug auf die Belegung von $\mathbf{P}$ erfüllt ist, da 'geben' in 'Hörer gibt Sprecher einen bestimmten Hammer' durch den Hörer erschlossen werden kann. Hinzukommt, da $B$ durch die Einstellungsbedingung gefordert wird, da B der vom Sprecher gewünschte Sachverhalt so beschaffen sein muB, da $B$ er für den Hörer realisierbar und für den Sprecher wünschenswert ist, und daB diese Bedingung in bezug auf den Sachverhalt 'Hörer gibt Sprecher einen bestimmten Hainmer' als erfüllt betrachtet werden können.

Der Außerungsbedeutung (48) wird aufgrund der Regel (44) die illokutive Funktion 'Hörer führt Sachverhalt aus' zugeordnet. Der kommunikative Sinn ergibt sich, wie

¿J Vgl. SCHEN K ER (1978). 
oben erwähnt, aus dem Bezug der Āußerungsbedeutung mit einer bestimmten illokutiven Funktion auf den Interaktionskontext. Für (48) kann danach folgender kommunikativer Sinn angenommen werden, setzt man voraus, da B die durch die illokutive Funktion fixierten Bedingungen und weitere kommunikative Anforderungen erfüllt sind.

(49) KS: Der Sprecher fordert den Hörer auf, ihm den Hammer zu geben.

\section{SchluBbemerkung}

Das zent rale Anliegen bestand darin, zu zeigen, worauf die semant ische Unbestimmtheit situationsgebundener, nicht durch den sprachlichen Kontext gestiitzter Ellipsen beruht, und wie sich die AuBerungsbedeutung und der kommunikative Sinn solcher Ellipsen aufgrund von Regeln und Prinzipien der Grammatik im Zusammenhang mit dem konzeptuellen und dem kommunikativen Kenntnissystem konstituieren. Die Untersuchungen beschränken sich dabei auf die Analyse eines typischen Beispiels und konzentrieren sich auf seine syntaktische und semantische Repräsentation sowie auf die Darstellung seiner AuBerungsbedeutung und seines kommunikativen Sinns. Dabei muBten aufgrund des diesbezïglichen Forschungsstandes ziemlich provisorische Voraussetzungen in Kauf genommen werden. Es ist deshalb gut möglich, da $B$ bei einer eventuellen Veränderung jener Voraussetzungen die hier erzielten Ergebnisse modifiziert werden müssen.

Die hauptsächlichen Ergebnisse dieses Artikels lassen sich in vier Punkten zusammenfassen: Es wurde erstens anhand eines Beispiels gezeigt, wie situative Ellipsen auf den verschiedenen Repräsentationsebenen der durch die Grammatik determinierten Laut-Bedeutungszuordnung strukturiert sind. Dabei wurde die von Brenwisch vertretene Annahme einer semantischen Formebene als Vermittlung zwischen der Ebene der Logischen Form und der konzeptuellen Formebene zugrunde gelegt. Zweitens wurde der Vorschlag unterbreitet, die semantische Unbestimmtheit situativer Ellipsen als das Ergebnis der semantischen Interpretation bestimmter syntaktisch leerer Kategorien als Argument- respektive Funktorvariablen anzusehen. Indem drittens die Grundmodi in die syntaktische und semantische Struktur situativer Ellipsen einbezogen worden sind, konnte die aus dem Grundmodus und dem propositionalen Gehalt zusammengesetzte semantische Form bezüglich ihrer Außerungsbedeutung nebst Einstellung und ihres kommunikativen Sinns untersucht werden. Dabei sollte versucht werden, bestimmte Bedingungen und Prinzipien zu zeigen, die die Wohlgeformtheit der Außerungsbedeutung und des kommunikativen Sinns festlegen. Viertens schließlich sollte dargelegt werden, da B situative Ellipsen sowohl syntaktische als auch semantische Besonderheiten im Vergleich zu Ellipsen aufweisen, die durch einen sprachlichen Kontext gestützt sind.

Es ist nicht zu übersehen, daß die hier in Angriff genommene Thematik einen großen Bogen spannt. Bestimmte Detailuntersuchungen mußten deshalb unberücksichtigt bleiben. Nicht unerwähnt soll bleiben, daB mit dem hier unterbreiteten Vorschlag zur syntaktischen und semantischen Repräsentation und zur Darstellung der Außerungsbedeutung und des kommunikativen Sinns viele offene Fragen, Probleme und alternative Vorschläge verknuipft sind. Sie wurden in geringem Maße in diesem Aufsatz und ausführlicher in Schwabe (1987) diskutiert oder zumindest formuliert. 


\section{Literatur}

BIERWISC, M. (1980): C'tterance meaning and mental states, In: F. KLIX and J. HoffMANy (eds.). Cognition and Memory, 55-64. Berlin.

- (1983 a): Basic Issues in the Development of Wordmeaning, in: Linguist ische Studien des ZISW der AdW der DDR, Reihe A, Heft 114.

- (1983b): Psychologische Aspekte der Semantik natürlicher Sprachen, in: W. Motsch, D. VIEH WEger (eds.), Richtungen der modernen Semantikforachung, Berlin.

- (1983()): Semantische und konzeptuelle Repräsentationen lexikalischer Einheilen, in: R. Rížı̇̌́cA and W. Motsck (eds.), Untersuchungen zur Semantik, Berlin ( = Studia grammatica XXII), 61-99.

- (1987): Dimensionsadjektive als strukturierender Ausschnitt des Sprachverhaltens, in: M. Bierwisch and E. Laxg (eds.), Grammatische und konzeptuelle Aspekte von Dimensionsadjektiven, Berlin (= Studia grammatica XXVI, XXVII).

Сномsky, N. (1981): Lectures on Government and Binding, Dordrecht, Holland, Cinnaminson, USA.

- (1986): Barriers, Cambridge, Massachusetts.

GrICE, P. (1968): The Logic of Conversation, Ms. Berkeley.

Grochowsw, M. (1985): Das Problem der Ellipse vom Slandpunkt der Satzgenerierungsregeln aus betrachtet, in: R. MEYER-HERMANN and H. Rteser (eds.), Ellipsen und fragmentarische Ausdrücke, Tübingen (=Linguistische Arbeiten 148/1,2).

Heidolph, K. E., Fläming, W., Moтsch, W. (1981): Grundzüge einer deutschen Grammatik, Berlin.

Jackendoff, R. S. (1977): X-Syntax: A study of phrase structure, Cambridge, Mass., London, England (= Linguistic Monograph Two).

Lana, E. (1983): Die logische Form eines Satzes als Gegenstand der linguistischen Semantik, in: W. Motsch and D. VIEHWEger (eds.), Richtungen der modernen Semantikforachung, Berlin.

Motsch, W., Рasch, R. (1987): Illokutive Handlungen, in: W. Motsch (ed.), Satz, Text, sprachliche Handlung, Berlin (= Studia grammatice XXV).

PAsch, R. (1983): Untersuchungen zu den Gebrauchsbedingungen der deutschen Kausalkonjunktionen da, denn und weil, in : Linguistische Studien des Zentralinstitu ts für Sprachwissenschaft der Akademie der Wissenschaften der DDR, Reihe A, Heft 104.

Pabch, R., Zmmmermann, I. (1983) : Die Rolle der Semantik in der Generativen Grammatik, in : W. Motsch und D. Vrenweger (eds.), Richtungen der modernen Semantikforschung, Berlin.

SCHENkER, W. (1978): Sprachliche Manieren. Eine sprachsoziologische Erhebung im Raum Trier und Eifel, Frankfurt a. M., Bern, Las Vegas.

Schwabe, K. (1987): Die Spezifik situativer Ellipsen, unv. Diss., Berlin.

Schopen, T. (1972): A Generative Theory of Ellipsis. A Consideration of the Linguistic Use of Silence, reproduced by the Indiana University Linguistic Club.

- (1983): Ellipsis as Grammatical Indeterminacy Foundation of Language 10.

Williams, E. (1977): Discourse and Logical Form, Linguistic Inquiry 8 (1).

ZnMmermavs, I. (1981): Uberlegungen zum Wesen von Kondensation und Ellipse, in: F. DaNES and D. VTERWearer (eds.), Satzsemantische Komponenten und Relationen in Text, in: Linguistica 1.

- (1984): Die Rolle des Lexikons in der Grammatik. Uberlegungen zu grammatischen Entwicklungen anhend des Passivs und der Subjekthebung in Deutschen, Deutsch als Fremdeprache 21 (1).

Zimmermans, I. (1985): Der syntaktische Parallelismus verbaler und adjektivischer Konstruktionen ( $\mathrm{Zu}$ einigen Grundfragen der X-Theorie), in: Linguistische Studien des ZISW der AdW der DDR, Reihe A, Heft 127.

- (1987): Komparationskonstruktionen, in: M. BIFrwisch and E. LANo (eds.), Grammatische und konzeptuelle Aspekte von Dimensionsadjektiven, Berlin (=Studia Grammatica XXVI, XXVII).

Dr. Kragtin SchwaBe, Akademie der. Wiseenschaften der DDR, Zentralinstitut für Sprachwissenschaft, Berlin

41 Z. Phonetlk (ZPSK) 41 (1088) 5 